\title{
On the Farrell-Jones Conjecture and its applications
}

\author{
Arthur Bartels, Wolfgang Lück and Holger Reich
}

\begin{abstract}
We present the status of the Farrell-Jones Conjecture for algebraic $K$-theory for a group $G$ and arbitrary coefficient rings $R$. We add new groups for which the conjecture is known to be true and study inheritance properties. We discuss new applications, focussing on the Bass Conjecture, the Kaplansky Conjecture and conjectures generalizing Moody's Induction Theorem. Thus we extend the class of groups for which these conjectures are known considerably.
\end{abstract}

Mathematics Subject Classification (2000). 19Dxx, 19A31,19B28

Keywords. Algebraic $K$-theory of group rings with arbitrary coefficients, Farrell-Jones Conjecture, Bass Conjecture, Kaplansky Conjecture, Moody's Induction Theorem.

\section{Introduction and statements of results}

0.1. Background. The Farrell-Jones Conjecture for algebraic K-theory predicts the structure of $K_{n}(R G)$ for a group $G$ and a ring $R$. There is also an $L$-theory version. For applications in topology and geometry the case $R=\mathbb{Z}$ is the most important one since many topological invariants of manifolds and $C W$-complexes such as the finiteness obstruction, the Whitehead torsion and the surgery obstruction take values in the algebraic $K$ - or $L$-theory of the integral group ring $\mathbb{Z} \pi$ of the fundamental group $\pi$. The Farrell-Jones Conjecture for $R=\mathbb{Z}$ implies several famous conjectures, e.g., the Novikov Conjecture, (in high dimensions) the Borel Conjecture, and the triviality of compact $h$-cobordisms with torsionfree fundamental group. On the other hand proofs of the Farrell-Jones Conjecture for certain groups often rely on working with integral coefficients since they are based on these geometric connections. This is the reason why more is known about the algebraic $K$ - and $L$-theory of $\mathbb{Z} G$ than of $\mathbb{C} G$ which is in some sense surprising since $\mathbb{C} G$ has better ring theoretic properties than $\mathbb{Z} G$. For the status of the Farrell-Jones Conjecture with coefficient in $\mathbb{Z}$ we refer for instance to [45, Sections 5.2 and 5.3].

Recently the geometric approaches have been generalized so far that they also apply to other coefficient rings than $\mathbb{Z}$ (see for instance Farrell-Linnell [30, BartelsReich [8], Bartels-Lück-Reich [6], Quinn [54]). This is interesting for algebraic and ring theoretic applications, where one would like to consider for example fields, rings of integers in algebraic number fields and integral domains. The purpose of 
this article is to describe the status of the Farrell-Jones Conjecture for algebraic $K$ theory for arbitrary coefficient rings and to discuss applications, for instance to the Bass Conjectures, the Kaplansky Conjecture, generalizations of Moody's Induction Theorem, Nil-groups and Fuglede-Kadison determinants.

0.2. Status of the Farrell-Jones Conjecture for algebraic $\boldsymbol{K}$ theory. There is a stronger version of the Farrell-Jones Conjecture, the so called Fibered Farrell-Jones Conjecture. The Fibered Farrell-Jones Conjecture does imply the Farrell-Jones Conjecture and has better inheritance properties than the Farrell-Jones Conjecture. We will give the precise technical formulations of these conjectures in Section 2, The original source for the (Fibered) Farrell-Jones Conjecture is the paper by Farrell-Jones [28, 1.6 on page 257 and 1.7 on page 262].

Ring will always mean associative ring with unit. It is not necessarily commutative. Fields are understood to be commutative unless they are called skew-fields.

One of the main results of this article is the next theorem whose proof will be given in Subsection 2.1.

Theorem 0.1. Let $R$ be a ring. Let $\mathcal{F} \mathcal{J}(R)$ be the class of groups which satisfy the Fibered Farrell-Jones Conjecture for algebraic $K$-theory with coefficients in $R$. Then

(i) Every word-hyperbolic group and every virtually nilpotent group belongs to $\mathcal{F} \mathcal{J}(R)$

(ii) If $G_{1}$ and $G_{2}$ belong to $\mathcal{F} \mathcal{J}(R)$, then $G_{1} \times G_{2}$ belongs to $\mathcal{F J}(R)$;

(iii) Let $\left\{G_{i} \mid i \in I\right\}$ be a directed system of groups (with not necessarily injective structure maps) such that $G_{i} \in \mathcal{F}$ for $i \in I$. Then $\operatorname{colim}_{i \in I} G_{i}$ belongs to $\mathcal{F} \mathcal{J}(R)$

(iv) If $H$ is a subgroup of $G$ and $G \in \mathcal{F} \mathcal{J}(R)$, then $H \in \mathcal{F} \mathcal{J}(R)$.

If one restricts to lower and middle $K$-theory for torsionfree groups and regular rings $R$, the Farrell-Jones Conjecture for algebraic $K$-theory reduces to the following easier to understand conclusions which are already very interesting in their own right.

Let $R$ be a ring and let $G$ be a group. Denote by $i: R \rightarrow R G$ the obvious inclusion. Sending $(g,[P]) \in G \times K_{0}(R)$ to the class of the $R G$-automorphism

$$
R[G] \otimes_{R} P \rightarrow R[G] \otimes_{R} P, \quad u \otimes x \mapsto u g^{-1} \otimes x
$$

defines a map $\Phi: G /[G, G] \otimes_{\mathbb{Z}} K_{0}(R) \rightarrow K_{1}(R G)$. Define the homomorphism

$$
A:=\Phi \oplus K_{1}(i):\left(G /[G, G] \otimes_{\mathbb{Z}} K_{0}(R)\right) \oplus K_{1}(R) \rightarrow K_{1}(R G) .
$$

Define $\mathrm{Wh}^{R}(G)$ to be the cokernel of $A$. If $\widetilde{K}_{0}(R)=0$ and the obvious map $R^{\times} \rightarrow K_{1}(R)$ is surjective, then $\mathrm{Wh}^{R}(G)$ coincides with $K_{1}(R G) /\langle(r \cdot g)| r \in$ $\left.R^{\times}, g \in G\right\rangle$. If $R=\mathbb{Z}$, then $\mathrm{Wh}^{\mathbb{Z}}(G)$ is the classical Whitehead group $\mathrm{Wh}(G)$ which appears for instance in the $s$-cobordism theorem. 
Theorem 0.2. Let $R$ be a regular ring. Suppose that $G$ is torsionfree and that the Farrell-Jones Conjecture for algebraic $K$-theory with coefficients in $R$ holds for $G$. Then

(i) $K_{n}(R G)=0$ for $n \leq-1$;

(ii) The change of rings map $K_{0}(R) \rightarrow K_{0}(R G)$ is bijective. In particular $\widetilde{K}_{0}(R G)$ is trivial if and only if $\widetilde{K}_{0}(R)$ is trivial;

(iii) The Whitehead group $\mathrm{Wh}^{R}(G)$ is trivial.

The proof of Theorem 0.2 can be found in 45, Conjecture 1.1 on page 652 , Conjecture 1.1 on page 657 and Corollary 2.3 on page 685].

In particular we get for $R=\mathbb{Z}$ that $\mathrm{Wh}(G)=0, \widetilde{K}_{0}(\mathbb{Z} G)=0$ and $K_{n}(\mathbb{Z} G)=0$ for $n \leq-1$ holds, if the torsionfree group $G$ satisfies the Farrell-Jones Conjecture with coefficient in $\mathbb{Z}$.

These vanishing results have important geometric consequences. Namely, let $G$ be a finitely presented group. Then $\mathrm{Wh}(G)$ vanishes if and only if every compact $h$ cobordism of dimension $\geq 6$ with $G$ as fundamental group is trivial, and $\widetilde{K}_{0}(\mathbb{Z} G)$ vanishes if and only if every finitely dominated $C W$-complex with $G$ as fundamental group is homotopy equivalent to a compact $C W$-complex. The vanishing of $\widetilde{K}_{0}(\mathbb{Z} G)$ implies that the group $G$ is already of type $\mathrm{FF}$ (which is sometimes also called type FL in the literature) if it is of type FP (see [16. Chapter VIII, Section 6]).

The conclusions appearing in Theorem 0.2 are known to be true for a torsionfree group $G$ provided that $G$ belongs to the class $\mathcal{F} \mathcal{J}(R)$ appearing in Theorem 0.1 , Examples are torsionfree subgroups of a finite product $\prod_{i=1}^{r} G_{i}$, where each group $G_{i}$ is word-hyperbolic. Subgroups of products of word-hyperbolic groups are studied for instance in [15].

More information about torsionfree groups $G$ for which Theorem 0.2 is true in the case $R=\mathbb{Z}$ can be found in [45, Theorem 5.5 on page 722]. There results due to Aravinda, Farrell, Hu, Jones, Linnell, and Roushon are listed.

\subsection{Homotopy $\boldsymbol{K}$-theory and rings with finite characteristic.}

Sometimes one wants to consider special rings or is interested in rational information only. Then the Fibered Farrell-Jones Conjecture is known to be true for more groups. This is illustrated by Theorem 0.3 below.

For the definition and basic properties of homotopy $K$-theory we refer to Weibel [67. For a positive integer $N$ let $\mathbb{Z}[1 / N]$ be the subring of $\mathbb{Q}$ consisting of rational numbers $m / n$ with $m, n \in \mathbb{Z}$ for which each prime dividing $n$ divides $N$. For an abelian group $A$ let $A[1 / N]$ be $A \otimes_{\mathbb{Z}} \mathbb{Z}[1 / N]$. A ring $R$ is said to be of finite characteristic if there is an integer $N \geq 2$ such that $N \cdot 1_{R}=0$. In this case the minimal positive integer with this property is called the characteristic of $R$.

The proof of the next theorem will be given in Subsection 2.3. The differences of the conclusions appearing in Theorem 0.1 and the following theorem are that now virtually nilpotent is replaced by elementary amenable in assertion (i) and 
that there are new assertions about extensions and actions on trees, namely assertions (v) and (vi). The extension result is intriguing since elementary amenable groups and word-hyperbolic groups form separate branches in Bridson's universe of groups (see [14]).

Theorem 0.3. Let $R$ be a ring. Consider the following assertions for a group $G$.

(KH) The group G satisfies the Fibered Farrell-Jones Conjecture for homotopy Ktheory with coefficients in $R$;

(FC) The ring $R$ has finite characteristic $N$. The Fibered Farrell-Jones Conjecture for algebraic $K$-theory for $G$ with coefficients in $R$ for both the families $\mathcal{F}$ in and $\mathcal{V C} y c$ is true after applying $-\otimes_{\mathbb{Z}} \mathbb{Z}[1 / N]$ to the assembly map.

Let $\mathcal{F J}_{K H}(R)$ be the class of groups for which assertion (KH) holds. If $R$ has finite characteristic, then let $\mathcal{F J}_{F C}(R)$ be the class of groups for which assertion $(F C)$ is true. Let $\mathcal{F}$ be $\mathcal{F} \mathcal{J}_{F C}(R)$ or $\mathcal{F} \mathcal{J}_{K H}(R)$. Then:

(i) Every word-hyperbolic and every elementary amenable group belongs to $\mathcal{F}$;

(ii) If $G_{1}$ and $G_{2}$ belong to $\mathcal{F}$, then $G_{1} \times G_{2}$ belongs to $\mathcal{F}$;

(iii) Let $\left\{G_{i} \mid i \in I\right\}$ be a directed system of groups (with not necessarily injective structure maps) such that $G_{i} \in \mathcal{F}$ for $i \in I$. Then $\operatorname{colim}_{i \in I} G_{i}$ belongs to $\mathcal{F}$;

(iv) If $H$ is a subgroup of $G$ and $G \in \mathcal{F}$, then $H \in \mathcal{F}$;

(v) Let $1 \rightarrow H \rightarrow G \rightarrow Q \rightarrow 1$ be an extension of groups such that $H$ is either elementary amenable or word-hyperbolic and $Q$ belongs to $\mathcal{F}$. Then $G$ belongs to $\mathcal{F}$;

(vi) Suppose that $G$ acts on a tree $T$. Assume that for each $x \in T$ the isotropy group $G_{x}$ belongs to $\mathcal{F}$. Then $G$ belongs to $\mathcal{F}$.

Moreover, if $R$ has finite characteristic then we have $\mathcal{F J}_{K H}(R) \subseteq \mathcal{F J}_{F C}(R)$.

Corollary 0.4. Let $R$ be a regular ring of finite characteristic $N$. Let $G$ be torsionfree. Suppose that $G$ belongs to the class $\mathcal{F J}_{F C}(R)$ defined in Theorem 0.3 . Then

(i) $K_{n}(R G)[1 / N]=0$ for $n \leq-1$;

(ii) The change of rings map induces a bijection $K_{0}(R)[1 / N] \rightarrow K_{0}(R G)[1 / N]$. In particular $\widetilde{K}_{0}(R G)[1 / N]$ is trivial if and only if $\widetilde{K}_{0}(R)[1 / N]$ is trivial;

(iii) $\mathrm{Wh}^{R}(G)[1 / N]$ is trivial.

The proof of Corollary 0.4 is analogous to the one of Theorem 0.2 Corollary 0.4 together with Theorem 0.3 substantially extends Theorem 1.1 of FarrellLinnell [30], where $\mathrm{Wh}^{F}(G) \otimes_{\mathbb{Z}} \mathbb{Q}=0$ is proven for $G$ a torsionfree elementary amenable group and $F$ a field of prime characteristic.

The vanishing of $\mathrm{Wh}^{\mathbb{F}_{p}}(G) \otimes_{\mathbb{Z}} \mathbb{Q}$ is needed in the definition of a $p$-adic logarithmic Fuglede-Kadison determinant for $G$ due to Deninger [22]. 
0.4. Induction from finite subgroups. The next result will be explained and proven in Section 3 .

Theorem 0.5. Let $G$ be a group. Then

(i) Let $R$ be a regular ring such that the order of any finite subgroup of $G$ is invertible in $R$, e.g., a field of characteristic zero. Suppose that $G$ satisfies the Farrell-Jones Conjecture with coefficients in $R$. Then the map given by induction from finite subgroups of $G$ (see (3.1))

$$
I(G, R): \underset{\operatorname{Cr}_{\mathcal{F} \text { in }}(G)}{\operatorname{colim}} K_{0}(R H) \rightarrow K_{0}(R G)
$$

is an isomorphism;

(ii) Let $D$ be a skew-field of characteristic $p$ for a prime number $p$. Suppose that $G$ satisfies the Farrell-Jones Conjecture with coefficients in $D$ after applying $-\otimes_{\mathbb{Z}} \mathbb{Z}[1 / p]$.

Then the map

$$
I(G, D)[1 / p]: \underset{\operatorname{Or}_{\mathcal{F} i n}(G)}{\operatorname{colim}} K_{0}(D H)[1 / p] \rightarrow K_{0}(D G)[1 / p]
$$

is an isomorphism.

Theorem 0.5 is an example of a result of the type that certain $K$-groups of a group ring are given by induction over finite subgroups. A prominent example is Moody's Induction Theorem (see [19, 49, [50]) which deals with the surjectivity of a corresponding map to $G_{0}(R G)$ instead of $K_{0}(R G)$ for virtually poly- $\mathbb{Z}$ groups $G$ and Noetherian rings $R$. If $R$ is regular and the order of any finite subgroup in the virtually poly- $\mathbb{Z}$ group $G$ is invertible in $R$, then $R G$ is regular and there is no difference between the $G$-theoretic and the $K$-theoretic statement. Thus Theorem 0.5 (i) is linked to Moody's induction theorem.

Every group in the family $\mathcal{F J}(R)$ appearing in Theorem 0.1 satisfies the assumptions of Theorem 0.5 (i)

Theorem 0.5 (ii) applies to every group $G$ which belongs to the family $\mathcal{F} \mathcal{J}_{F C}(D)$ appearing in Theorem 0.3 . Thus we have substantially generalized Theorem 1.2 of Farrell-Linnell [30], where the surjectivity of the map

$$
I(G, F) \otimes_{\mathbb{Z}} \mathbb{Q}: \operatorname{colim}_{\operatorname{Or}_{\mathcal{F i n}}(G)} K_{0}(F H) \otimes_{\mathbb{Z}} \mathbb{Q} \rightarrow K_{0}(F G) \otimes_{\mathbb{Z}} \mathbb{Q}
$$

is proven for elementary amenable groups $G$ and fields $F$ of prime characteristic.

0.5. Bass Conjectures. The following conjecture is due to Bass [11, 4.5].

Conjecture 0.6 (Bass Conjecture for commutative integral domains). Let $R$ be a commutative integral domain and let $G$ be a group. Let $g \in G$. Suppose that either the order $|g|$ is infinite or that the order $|g|$ is finite and not invertible in $R$.

Then for every finitely generated projective $R G$-module the value of its HattoriStallings rank $\operatorname{HS}_{R G}(P)$ at $(g)$ (see (4.30) is zero. 
The Bass Conjecture 0.6 can be interpreted topologically. Namely, the Bass Conjecture 0.6 is true for a finitely presented group $G$ in the case $R=\mathbb{Z}$ if and only if every homotopy idempotent selfmap of an oriented smooth closed manifold whose dimension is greater than 2 and whose fundamental group is isomorphic to $G$ is homotopic to one that has precisely one fixed point (see [13). The Bass Conjecture 0.6 for $G$ in the case $R=\mathbb{Z}$ (or $R=\mathbb{C}$ ) also implies for a finitely dominated $C W$-complex with fundamental group $G$ that its Euler characteristic agrees with the $L^{2}$-Euler characteristic of its universal covering (see 24]).

The next results follows from the argument in [30, Section 5].

Theorem 0.7. Let $G$ be a group. Suppose that

$$
I(G, F) \otimes_{\mathbb{Z}} \mathbb{Q}: \operatorname{colim}_{\operatorname{Or}_{\mathcal{F} i n}(G)} K_{0}(F H) \otimes_{\mathbb{Z}} \mathbb{Q} \rightarrow K_{0}(F G) \otimes_{\mathbb{Z}} \mathbb{Q}
$$

is surjective for all fields $F$ of prime characteristic.

Then the Bass Conjecture 0.6 is satisfied for every commutative integral domain $R$.

Hence by Theorem 0.5 the Bass Conjecture for commutative integral domains 0.6 is true for every group $G$ which lies in the class $\mathcal{F J}_{F C}(F)$ for all fields $F$ of prime characteristic. The case of elementary amenable groups has already been treated in [30, Theorem 1.6]. The Bass Conjecture 0.6 has been proved by Bass 11, Proposition 6.2 and Theorem 6.3] for $R=\mathbb{C}$ and $G$ a linear group, by Linnell [38, Lemma 4.1] for $|g|<\infty$ and $R=\mathbb{Z}$, and by Eckmann [23, Theorem 3.3] for $R=\mathbb{Q}$ provided that $G$ has at most cohomological dimension 2 over $\mathbb{Q}$. Further results are proved by Emmanouil [27].

Here is another version of the Bass Conjecture.

Conjecture 0.8 (Bass Conjecture for fields of characteristic zero as coefficients). Let $F$ be a field of characteristic zero and let $G$ be a group. The Hattori-Stallings homomorphism (see (4.3)) induces an isomorphism

$$
\mathrm{HS}_{F G}: K_{0}(F G) \otimes_{\mathbb{Z}} F \rightarrow \operatorname{class}_{F}(G)_{f} .
$$

For a field $F$ of characteristic zero Conjecture 0.8 obviously implies Conjecture 0.6. The proof of the next result will be given in Section 4

Theorem 0.9. Let $F$ be a field of characteristic zero and let $G$ be a group. If $G$ satisfies the Farrell-Jones Conjecture with coefficients in $F$, then $G$ satisfies the Bass-Conjecture 0.8 for $F$.

In particular the Bass Conjecture for a field $F$ of characteristic zero as coefficients 0.8 is true for all groups in the class $\mathcal{F} \mathcal{J}(F)$ by Theorem 0.1 .

Berrick-Chatterji-Mislin [12] prove that a group $G$ satisfies the Bass Conjecture 0.8 for $F=\mathbb{C}$ and the Bass Conjecture 0.6 for integral domains for $R=\mathbb{Z}$ if $G$ satisfies the Bost Conjecture. Because the Bost Conjecture is known for many groups, this is also true for the Bass Conjecture for $F=\mathbb{C}$. Since the Bost Conjecture deals with $l^{2}$-spaces, this strategy can only work for subrings of $\mathbb{C}$. 
The following result describes another conclusion of the Farrell-Jones Conjecture, which is in the spirit of the Bass Conjecture. It is hence true for all groups in the family $\mathcal{F} \mathcal{J}(R)$.

Theorem 0.10. Let $G$ be a group. Let $R$ be a commutative integral domain with quotient field $F$ such that no prime divisor of the order of a finite subgroup of $G$ is invertible in $R$. (An example is $R=\mathbb{Z}$ and $F=\mathbb{Q}$.) Suppose that $G$ satisfies the Farrell-Jones Conjecture for algebraic $K$-theory with coefficients in $R$.

Then the change of rings homomorphisms

$$
K_{0}(R G) \otimes_{\mathbb{Z}} \mathbb{Q} \rightarrow K_{0}(F G) \otimes_{\mathbb{Z}} \mathbb{Q}
$$

agrees with the composite

$$
K_{0}(R G) \otimes_{\mathbb{Z}} \mathbb{Q} \rightarrow K_{0}(R) \otimes_{\mathbb{Z}} \mathbb{Q} \rightarrow K_{0}(F) \otimes_{\mathbb{Z}} \mathbb{Q} \rightarrow K_{0}(F G) \otimes_{\mathbb{Z}} \mathbb{Q}
$$

where the three maps come from the change of ring homomorphisms associated to the augmentation $R G \rightarrow R$, the inclusion $R \rightarrow F$ and the inclusion $F \rightarrow F G$. In particular the homomorphism

$$
\widetilde{K}_{0}(R G) \otimes_{\mathbb{Z}} \mathbb{Q} \rightarrow \widetilde{K}_{0}(F G) \otimes_{\mathbb{Z}} \mathbb{Q}
$$

is trivial.

(We remind the reader, that $\widetilde{K}_{0}(R G)$ and $\widetilde{K}_{0}(F G)$ respectively is the cokernel of the canonical map $K_{0}(\mathbb{Z}) \rightarrow K_{0}(R G)$ and the canonical map $K_{0}(\mathbb{Z}) \rightarrow K_{0}(F G)$ respectively.)

If $G$ is finite, $G$ satisfies the Farrell-Jones Conjecture for algebraic $K$-theory with coefficients in $R$ for trivial reasons and hence Theorem 0.10 reduces to a Theorem of Swan (see [63, Theorem 8.1], [11, Corollary 4.2]).

The conclusion of Theorem 0.10 is related to the theorem that for every group $G$ the change of rings homomorphism $\widetilde{K}_{0}(\mathbb{Z} G) \rightarrow \widetilde{K}_{0}(\mathcal{N}(G))$ is trivial, where $\mathcal{N}(G)$ is the group von Neumann algebra (see [41, Theorem 9.62 on page 363], [59]).

\subsection{The Kaplansky Conjecture.}

Conjecture 0.11 (Kaplansky Conjecture). Let $R$ be an integral domain and let $G$ be a torsionfree group. Then all idempotents of $R G$ are trivial, i.e., equal to 0 or 1 .

In the next theorem we will use the notion of a sofic group that was introduced by Gromov and originally called subamenable group. Every residually amenable group is sofic but the converse is not true. The class of sofic groups is closed under taking subgroups, direct products, free amalgamated products, colimits and inverse limits, and, if $H$ is a sofic normal subgroup of $G$ with amenable quotient $G / H$, then $G$ is sofic. For more information about the notion of a sofic group we refer to [26.

The proof and further explanations of the next theorem will be given in Section 5. 
Theorem 0.12. Let $G$ be a group. Let $R$ be a ring whose idempotents are all trivial. Suppose that

$$
K_{0}(R) \otimes_{\mathbb{Z}} \mathbb{Q} \rightarrow K_{0}(R G) \otimes_{\mathbb{Z}} \mathbb{Q}
$$

is an isomorphism.

Then the Kaplansky Conjecture holds for $R$ and $G$ if one of the following conditions is satisfied:

(i) $R G$ is stably finite;

(ii) $R$ is a field of characteristic zero;

(iii) $R$ is a skew-field and $G$ is sofic.

Next we discuss some special cases of Theorem 0.12 . Notice that we get assertions also for skew-fields and not only for fields as coefficients.

Theorem 0.2 and Theorem 0.12 imply that for a skew-field $D$ of characteristic zero and a torsionfree group $G$ belonging to the class of groups $\mathcal{F} \mathcal{J}(D)$ defined in Theorem 0.1 the Kaplansky Conjecture 0.11 is true for $D G$, provided that $D$ is commutative or that $G$ is sofic.

Suppose that $D$ is a skew-field of prime characteristic $p$, all finite subgroups of $G$ are $p$-groups and $G$ belongs to the class $\mathcal{F J}_{F C}(D)$ defined in Theorem 0.3 . Then $K_{0}(D) \otimes_{\mathbb{Z}} \mathbb{Q} \stackrel{\cong}{\rightarrow} K_{0}(D G) \otimes_{\mathbb{Z}} \mathbb{Q}$ is an isomorphism by Theorem 0.5)(ii) since for a finite $p$-group $H$ the group ring $D H$ is a local ring and hence $\widetilde{K}_{0}(D H)=0$. If we furthermore assume that $G$ is sofic, then Theorem 0.12 implies that all idempotents in $D G$ are trivial. This has already been proved in the case where $G$ is elementary amenable and $D$ is commutative by Farrell-Linnell [30, Theorem 1.7].

To the authors' knowledge there is no example of a group which is not sofic and of a group which is word-hyperbolic and not residually finite in the literature. So it is conceivable that all word-hyperbolic groups are sofic.

Next we mention some results of others.

Let $F$ be a field of characteristic zero and $u=\sum_{g \in G} x_{g} \cdot g \in K[G]$ be an idempotent. Let $K$ be the finitely generated field extension of $\mathbb{Q}$ given by $K=$ $\mathbb{Q}\left(x_{g} \mid g \in G\right)$. Obviously $u \in K G$ is an idempotent. There exists an embedding of $K$ in $\mathbb{C}$. Hence all idempotents in $F G$ are non-trivial if all idempotents in $\mathbb{C} G$ are trivial.

The Kadison Conjecture says that all idempotents in the reduced group $C^{*}$ algebra $C_{r}^{*}(G)$ of a torsionfree group are trivial. Hence the Kadison Conjecture implies the Kaplansky Conjecture for all fields of characteristic zero. The Kadison Conjecture follows from the Baum-Connes Conjecture (as explained for instance in [42, 45, 1.8.1 and 1.8.2]). Hence a torsionfree group $G$ satisfies the Kaplansky Conjecture for all fields of characteristic zero, if it satisfies the Baum-Connes Conjecture. For a survey of groups satisfying the Baum-Connes Conjecture we refer to [45, Sections 5.1 and 5.3]. We mention that subgroups of word-hyperbolic groups satisfy the Baum-Connes Conjecture by a result of Mineyev-Yu 48, Theorem 20] based on the work of Lafforgue [37] (see also [61]). A proof of the Kadison Conjecture for a torsionfree word-hyperbolic group using cyclic homology is given 
by Puschnigg [52. Notice that all these analytic methods do only work for fields of characteristic zero and cannot be extended to skew-fields or fields of prime characteristic.

Formanek [32] (see also [17, Lemma 4.1 and Proposition 4.2]) has shown that all idempotents of $F G$ are trivial provided that $F$ is a field of prime characteristic $p$, the group $G$ contains no $p$-torsion and there do not exist an element $g \in G, g \neq 1$ and an integer $k \geq 1$ such that $g$ and $g^{p^{k}}$ are conjugate, or, provided that $F$ is a field of characteristic zero and there are infinitely many primes $p$ for which there do not exist an element $g \in G, g \neq 1$ and an integer $k \geq 1$ such that $g$ and $g^{p^{k}}$ are conjugate. Torsionfree word-hyperbolic groups satisfy these conditions. Hence Formanek's results imply that all idempotents in $F G$ are trivial if $G$ is torsionfree word-hyperbolic and $F$ is a field.

Delzant 21] has proven the Kaplansky Conjecture 0.11 for all integral domains $R$ for a torsionfree word-hyperbolic group $G$ provided that $G$ admits an appropriate action with large enough injectivity radius. Delzant actually deals with zero-divisors and units as well.

0.7. Homotopy invariance of $\boldsymbol{L}^{\mathbf{2}}$-torsion. The following conjecture for a group $G$ is stated and explained in Lück [41, Conjecture 3.94 (1) on page 163].

Conjecture 0.13. Define the homomorphism

$$
\Phi=\Phi^{G}: \operatorname{Wh}(G) \rightarrow \mathbb{R}
$$

by sending the class $[A]$ of an invertible matrix $A \in G L_{n}(\mathbb{Z} G)$ to $\ln \left(\operatorname{det}\left(r_{A}^{(2)}\right)\right)$, where $\operatorname{det}\left(r_{A}^{(2)}\right)$ is the Fuglede-Kadison determinant of the G-equivariant bounded operator $l^{2}(G)^{n} \rightarrow l^{2}(G)^{n}$ given by right multiplication with $A$.

Then $\Phi$ is trivial.

It is important because of the following conclusion explained in 41, Theorem 3.94 (1)) on page 161]: If $X$ and $Y$ are det- $L^{2}$-acyclic finite $G-C W$-complexes, which are $G$-homotopy equivalent, then their $L^{2}$-torsions agree:

$$
\rho^{(2)}(X ; \mathcal{N}(G))=\rho^{(2)}(Y ; \mathcal{N}(G)) .
$$

Theorem 0.14. Suppose that $G$ satisfies the Farrell-Jones Conjecture for algebraic $K$-theory with coefficients in $\mathbb{Z}$. Then $G$ satisfies Conjecture 0.13 .

We will omit the proof of Theorem 0.14 since it is similar to the one of Theorem 0.10 using the fact that for a finite group $H$ we have $\widetilde{K}_{0}(\mathbb{Z} H) \otimes_{\mathbb{Z}} \mathbb{Q}=0$ and Conjecture 0.13 is true for finite groups for elementary reasons.

Let $G$ be a torsionfree word-hyperbolic group. Suppose that its $L^{2}$-Betti numbers $b_{p}^{(2)}(G)$ are trivial for all $p \geq 0$ and that $G$ is of det $\geq 1$-class. (If $G$ is residually finite, it is of det $\geq 1$ - class.) Choose a cocompact model for $E G$. Then we can define the $L^{2}$-torsion of $G$

$$
\rho^{(2)}(G):=\rho^{(2)}(E G ; \mathcal{N}(G)) \in \mathbb{R} .
$$


This is indeed independent of the choice of a cocompact model for $E G$ and hence depends only on $G$ by Theorem 0.1 and Theorem 0.14 . If $M$ is a closed hyperbolic manifold of dimension $2 n+1$, then its fundamental group $\pi=\pi_{1}(M)$ satisfies all these assumptions and there exists a number $C_{n}>0$ depending only on $n$ such that $C_{n} \cdot(-1)^{n} \cdot \rho^{(2)}(\pi)$ is the volume of $M$. Hence $\rho^{(2)}(G)$ can be viewed as a kind of volume of a word-hyperbolic group $G$, provided $G$ satisfies the above assumptions.

0.8. Searching for counterexamples. There is no group known for which the Farrell-Jones Conjecture, the Fibered Farrell-Jones Conjecture or the Baum-Connes Conjecture is false. However, Higson, Lafforgue and Skandalis 35, Section 7] construct counterexamples to the Baum-Connes-Conjecture with coefficients, actually with a commutative $C^{*}$-algebra as coefficients. They describe precisely what properties a group $\Gamma$ must have so that it does not satisfy the Baum-Connes Conjecture with coefficients. Gromov [33] describes the construction of such a group $\Gamma$ as a colimit over a directed system of groups $\left\{G_{i} \mid i \in I\right\}$ such that each $G_{i}$ is word-hyperbolic. We conclude from Bartels-Echterhoff-Lück [3] and Theorem 0.1 that the Fibered Farrell-Jones Conjecture and the Bost Conjecture do hold for $\Gamma$.

0.9. Nil-groups. In Section 6 we discuss some consequences for Nil-groups in the sense of Bass and Waldhausen and for the passage from algebraic $K$-theory to homotopy $K$-theory. There we will prove the following application of Theorem 0.1 (i) to Waldhausen's Nil-groups.

Theorem 0.15. Let $G, H$ and $K$ be finite groups. Let $C:=\mathbb{Z}[K]$.

(i) Let $\alpha: K \rightarrow G$ and $\beta: K \rightarrow H$ be injective group homeomorphisms. Consider the $C$ bimodules $A^{\prime}:=\mathbb{Z}[G-\alpha(K)]$ and $B^{\prime}:=\mathbb{Z}[H-\beta(K)]$. Then

$$
\mathrm{Nil}_{n}\left(C ; A^{\prime}, B^{\prime}\right) \otimes_{\mathbb{Z}} \mathbb{Q}=0
$$

(ii) Let $\alpha: K \rightarrow G$ and $\beta: K \rightarrow G$ be injective group homeomorphisms. Let $A^{\prime}:=\mathbb{Z}[G-\alpha(K)], A^{\prime \prime}:=\mathbb{Z}[H-K]$ and $A:=\mathbb{Z}[G]$. Then

$$
\operatorname{Nil}_{n}\left(C ;{ }_{\alpha} A_{\alpha}^{\prime},{ }_{\beta} A_{\beta}^{\prime \prime},{ }_{\beta} A_{\alpha},{ }_{\alpha} A_{\beta}\right) \otimes_{\mathbb{Z}} \mathbb{Q}=0,
$$

where we used the lower indices to indicate the relevant $C$-bimodule structures.

0.10. The Farrell-Jones Conjecture for L-theory. In Section 7 we briefly explain some results about the $L$-theoretic version of the Farrell-Jones Conjecture. 


\section{Inheritance Properties of the (Fibered) Isomor- phism Conjecture}

In this section we formulate a (Fibered) Isomorphism Conjecture for a given equivariant homology theory and a family of groups. In this general setting we study the behavior of this Fibered Isomorphism Conjecture under directed colimits and extensions. The Farrell-Jones Conjecture is a special case, one has to choose a specific equivariant homology theory and a specific family of subgroups. The payoff of this general setting is that some of the proofs become easier and more transparent and that it applies to other related Isomorphism Conjectures such as the FarrellJones Conjecture for L-theory, the Baum-Connes Conjecture, the Bost Conjecture and other types of Isomorphism Conjectures predicting the bijectivity of certain assembly maps.

For this section we fix the following data

- a discrete group $G$;

- an equivariant homology theory $\mathcal{H}_{*}^{\text {? }}$ with values in $\Lambda$-modules;

- a class of groups $\mathcal{C}$ closed under isomorphisms, taking subgroups and taking quotients, e.g., the family $\mathcal{F}$ in of finite groups and the family $\mathcal{V C}$ yc of virtually cyclic groups. For a group $G$ we denote by $\mathcal{C}(G)$ the family of subgroups of $G$ which belong to $\mathcal{C}$.

Here equivariant homology theory with values in $\Lambda$-modules for a commutative ring $\Lambda$ satisfying the disjoint union axiom is understood in the sense of [40, Section 1] with one important modification: We require that for every group homomorphism $\alpha: H \rightarrow K$ we get a natural transformation

$$
\operatorname{ind}_{\alpha}: \mathcal{H}_{*}^{H}(X) \rightarrow \mathcal{H}_{n}^{K}\left(\operatorname{ind}_{\alpha} X\right)
$$

satisfying the obvious variations of the axioms a.) Compatibility with the boundary operator b.) Naturality and c.) Compatibility with conjugation, but the map ind $\alpha$ is only required to be an isomorphism in the case, where $X=\mathrm{pt}$ and $\alpha$ is injective. This implies that $\operatorname{ind}_{\alpha}$ is bijective for a $G$-CW-complex $X$ if the kernel of $\alpha$ acts freely on $X$ (see [3, Lemma 1.5]). Every Groupoids-spectrum gives an equivariant homology theory with values in $\mathbb{Z}$-modules in the sense above (see [45, 6.5]). In particular we get an equivariant homology theory with values in $\mathbb{Z}$-modules in the sense above for algebraic $K$-theory (see [20, Section 2], 45, Theorem 6.1]). If $\mathcal{H}_{*}^{\text {? }}$ is an equivariant homology theory with values in $\mathbb{Z}$-modules, then $\mathcal{H}_{*}^{?} \otimes_{\mathbb{Z}} \Lambda$ is an equivariant homology theory with values in $\Lambda$-modules for $\mathbb{Z} \subseteq \Lambda \subseteq \mathbb{Q}$.

Notice that this is one of the key differences between the Farrell-Jones Conjecture for algebraic $K$ - and $L$-theory and the Baum-Connes Conjecture for topological $K$-theory of reduced group $C^{*}$-algebras. In the latter case induction is only defined if the kernel of the group homomorphism acts freely because the corresponding spectrum lives over Groupoids ${ }^{\text {inj }}$ and not over Groupoids as in the Farrell-Jones setting (see [45, 6.5]). 
1.1. The Fibered Isomorphism Conjecture for equivariant homology theories. A family of subgroups of $G$ is a collection of subgroups of $G$ which is closed under conjugation and taking subgroups. Let $E_{\mathcal{F}}(G)$ be the classifying space associated to $\mathcal{F}$. It is uniquely characterized up to $G$-homotopy by the properties that it is a $G$-CW-complex and that $E_{\mathcal{F}}(G)^{H}$ is contractible if $H \in \mathcal{F}$ and is empty if $H \notin \mathcal{F}$. For more information about these spaces $E_{\mathcal{F}}(G)$ we refer to the survey article [44. Given a group homomorphism $\phi: K \rightarrow G$ and a family $\mathcal{F}$ of subgroups of $G$, define the family $\phi^{*} \mathcal{F}$ of subgroups of $K$ by

$$
\phi^{*} \mathcal{F}=\{H \subseteq K \mid \phi(H) \in \mathcal{F}\} .
$$

If $\phi$ is an inclusion of a subgroup, we also write $\left.\mathcal{F}\right|_{K}$ instead of $\phi^{*} \mathcal{F}$.

Definition 1.1 ((Fibered) Isomorphism Conjecture for $\left.\mathcal{H}_{*}^{?}\right)$. A group $G$ together with a family of subgroups $\mathcal{F}$ satisfies the Isomorphism Conjecture for $\mathcal{H}_{*}^{\text {? }}$ if the projection pr: $E_{\mathcal{F}}(G) \rightarrow$ pt to the one-point-space pt induces an isomorphism

$$
\mathcal{H}_{n}^{G}(\mathrm{pr}): \mathcal{H}_{n}^{G}\left(E_{\mathcal{F}}(G)\right) \stackrel{\cong}{\rightrightarrows} \mathcal{H}_{n}^{G}(\mathrm{pt})
$$

for $n \in \mathbb{Z}$.

The pair $(G, \mathcal{F})$ satisfies the Fibered Isomorphism Conjecture for $\mathcal{H}_{*}^{\text {? }}$ if for every group homomorphism $\phi: K \rightarrow G$ the pair $\left(K, \phi^{*} \mathcal{F}\right)$ satisfies the Isomorphism Conjecture.

We mostly work with a fixed equivariant homology theory $\mathcal{H}_{*}^{?}$ and hence we will often omit it in the statements.

The following results are proven in [4, Lemma 1.6] and [5, Lemma 1.2 and Theorem 2.4]

Lemma 1.2. Let $G$ be a group and let $\mathcal{F} \subset \mathcal{G}$ be families of subgroups of $G$. Suppose that $(G, \mathcal{F})$ satisfies the Fibered Isomorphism Conjecture.

Then $(G, \mathcal{G})$ satisfies the Fibered Isomorphism Conjecture.

Lemma 1.3. Let $\phi: K \rightarrow G$ be a group homomorphism and let $\mathcal{F}$ be a family of subgroups. If $(G, \mathcal{F})$ satisfies the Fibered Isomorphism Conjecture, then $\left(K, \phi^{*} \mathcal{F}\right)$ satisfies the Fibered Isomorphism Conjecture.

Theorem 1.4 (Transitivity Principle). Let $\mathcal{F} \subseteq \mathcal{G}$ be two families of subgroups of $G$. Assume that for every element $H \in \mathcal{G}$ the group $H$ satisfies the (Fibered) Isomorphism Conjecture for $\left.\mathcal{F}\right|_{H}$.

Then $(G, \mathcal{G})$ satisfies the (Fibered) Isomorphism Conjecture if and only if $(G, \mathcal{F})$ satisfies the (Fibered) Isomorphism Conjecture.

The next lemma follows from Lemma 1.3 applied to the inclusion $H \rightarrow G$ since $\mathcal{C}(H)=\left.\mathcal{C}(G)\right|_{H}$.

Lemma 1.5. Suppose that the Fibered Isomorphism Conjecture holds for $(G, \mathcal{C}(G))$. Let $H \subseteq G$ be a subgroup.

Then the Fibered Isomorphism Conjecture holds for $(H, \mathcal{C}(H))$. 
1.2. Colimits over directed systems of groups. We collect some basic facts about the behavior of the Fibered Isomorphism Conjecture under directed colimits.

We consider a directed set $I$ and a directed system of groups $\left\{G_{i} \mid i \in I\right\}$. The structure maps $\phi_{i, j}: G_{i} \rightarrow G_{j}$ for $i, j \in I$ with $i \leq j$ are not required to be injective. Let $\operatorname{colim}_{i \in I} G_{i}$ be the colimit. Denote by $\psi_{i}: G_{i} \rightarrow G$ the structure maps of the colimit for $i \in I$.

We say that $G$ is the directed union of the subgroups $\left\{G_{i} \mid i \in I\right\}$ if $I$ is a directed set and $\left\{G_{i} \mid i \in I\right\}$ is a directed system of subgroups, directed by inclusion, such that $G=\bigcup_{i \in I} G_{i}$. This is essentially the same as a directed system of groups such that all structure maps $\phi_{i, j}$ are inclusions of groups and $G=\operatorname{colim}_{i \in I} G_{i}$. For a group homomorphism $\psi: G^{\prime} \rightarrow G$ define the $\Lambda$-map

$$
\alpha_{n}(\psi): \mathcal{H}_{n}^{G^{\prime}}(\mathrm{pt}) \rightarrow \mathcal{H}_{n}^{G}(\mathrm{pt})
$$

as the composition of $\operatorname{ind}_{\psi}$ with the map induced by the projection $\psi^{*}$ pt $\rightarrow$ pt of $G$-spaces.

The next definition is an extension of [4, Definition 3.1].

Definition 1.6 ((Strongly) Continuous equivariant homology theory). An equiv-

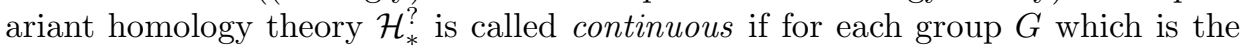
directed union of subgroups $\left\{G_{i} \mid i \in I\right\}$ the $\Lambda$-map

$$
\operatorname{colim}_{i \in I} \alpha_{n}\left(G_{i} \rightarrow G\right): \operatorname{colim}_{i \in I} \mathcal{H}_{n}^{G_{i}}(\mathrm{pt}) \rightarrow \mathcal{H}_{n}^{G}(\mathrm{pt})
$$

is an isomorphism for every $n \in \mathbb{Z}$.

An equivariant homology theory $\mathcal{H}_{*}^{?}$ is called strongly continuous if for each directed system of groups $\left\{G_{i} \mid i \in I\right\}$ with $G=\operatorname{colim}_{i \in I} G_{i}$ the $\Lambda$-map

$$
\underset{i \in I}{\operatorname{colim}} \alpha_{n}\left(\psi_{i}\right): \operatorname{colim}_{i \in I} \mathcal{H}_{n}^{G_{i}}(\mathrm{pt}) \rightarrow \mathcal{H}_{n}^{G}(\mathrm{pt})
$$

is an isomorphism for every $n \in \mathbb{Z}$.

The next theorem generalizes the result of Farrell-Linnell [29, Theorem 7.1] to a more general setting about equivariant homology theories as developed in Bartels-Lück [4. Its proof can be found in [3, Theorems 3.4 and 4.6].

Theorem 1.7. (i) Let $G$ be the directed union $G=\bigcup_{i \in I} G_{i}$ of subgroups $G_{i}$ Suppose that $\mathcal{H}_{*}^{?}$ is continuous and that the (Fibered) Isomorphism Conjecture is true for $\left(G_{i}, \mathcal{C}\left(G_{i}\right)\right)$ for all $i \in I$.

Then the (Fibered) Isomorphism Conjecture is true for $(G, \mathcal{C}(G))$;

(ii) Let $\left\{G_{i} \mid i \in I\right\}$ be a directed system of groups. Put $G=\operatorname{colim}_{i \in I} G_{i}$. Suppose that $\mathcal{H}_{*}^{?}$ is strongly continuous and that the Fibered Isomorphism Conjecture is true for $\left(G_{i}, \mathcal{C}\left(G_{i}\right)\right)$ for all $i \in I$.

Then the Fibered Isomorphism Conjecture is true for $(G, \mathcal{C}(G))$. 
1.3. Extensions. For the remainder of this section fix the following data:

- a discrete group $G$;

- an equivariant homology theory $\mathcal{H}_{*}^{\text {? }}$ with values in $\Lambda$-modules;

- a class of groups $\mathcal{C}$ closed under isomorphisms, taking subgroups and taking quotients, e.g., $\mathcal{F}$ in or $\mathcal{V C y c}$;

- an exact sequence of groups $1 \rightarrow K \stackrel{i}{\rightarrow} G \stackrel{p}{\rightarrow} Q \rightarrow 1$.

We want to investigate the inheritance properties of the (Fibered) Isomorphism Conjecture 1.1 under exact sequences.

Lemma 1.8. Suppose that the Fibered Isomorphism Conjecture holds for $(Q, \mathcal{C}(Q))$ and for every $H \in p^{*} \mathcal{C}(Q)$ the Isomorphism Conjecture is true for $(H, \mathcal{C}(H))$.

Then the Isomorphism Conjecture is true for $(G, \mathcal{C}(G))$.

Proof. Since the Fibered Isomorphism Conjecture holds for $(Q, \mathcal{C}(Q))$ by assumption, the Isomorphism Conjecture holds for $\left(G, p^{*} \mathcal{C}(Q)\right)$. We have to show that the Isomorphism Conjecture holds for $(G, \mathcal{C}(G))$. But this follows from the Transitivity Principle 1.4

Lemma 1.9. Suppose that the Fibered Isomorphism Conjecture holds for $(Q, \mathcal{C}(Q))$. Then the following assertions are equivalent.

(i) The Fibered Isomorphism Conjecture is true for $\left(p^{-1}(H), \mathcal{C}\left(p^{-1}(H)\right)\right)$ for every $H \in \mathcal{C}(Q)$;

(ii) The Fibered Isomorphism Conjecture is true for $(G, \mathcal{C}(G))$.

Proof. (ii) $\Rightarrow$ (i) This follows from Lemma[1.3] applied to the inclusion $p^{-1}(H) \rightarrow G$. (i) $\Rightarrow$ (ii) Let $q: L \rightarrow G$ be a group homomorphism. We have to show that $\left(L, q^{*} \mathcal{C}(G)\right)$ satisfies the Isomorphism Conjecture. Since $(Q, \mathcal{C}(Q))$ satisfies the Fibered Isomorphism Conjecture, we conclude that $\left(L, q^{*} p^{*} \mathcal{C}(Q)\right)$ satisfies the Isomorphism Conjecture. Because of the Transitivity principle 1.4 it remains to show for any $H \subseteq L$ for which there exists $V \in \mathcal{C}(Q)$ with $q(H) \subseteq p^{-1}(V)$ that $\left(H,\left.\left(q^{*} \mathcal{C}(G)\right)\right|_{H}\right)$ satisfies the Isomorphism Conjecture. This follows from the assumption that $\left(p^{-1}(V), \mathcal{C}\left(p^{-1}(V)\right)\right)$ satisfies the Fibered Isomorphism Conjecture since the families $\left.\left(q^{*} \mathcal{C}(G)\right)\right|_{H}$ and $\left(\left.q\right|_{H}\right)^{*} \mathcal{C}\left(p^{-1}(V)\right)$ coincide.

Lemma 1.10. Suppose that $p^{-1}(H)$ belongs to $\mathcal{C}(G)$ if $H \in \mathcal{C}(Q)$. Then $(G, \mathcal{C}(G))$ satisfies the Fibered Isomorphism Conjecture, if $(Q, \mathcal{C}(Q))$ satisfies the Fibered Isomorphism Conjecture.

Proof. This follows from Lemma 1.3 since $p^{*} \mathcal{C}(Q)=\mathcal{C}(G)$.

Lemma 1.10 is interesting in the case, where $\mathcal{C}$ is $\mathcal{F}$ in or $\mathcal{V} \mathcal{C y c}$ and $K$ is finite. 
Lemma 1.11. (i) Suppose that $H_{1} \times H_{2}$ belongs to $\mathcal{C}$ if $H_{1}$ and $H_{2}$ belong to $\mathcal{C}$. Then $\left(G_{1} \times G_{2}, \mathcal{C}\left(G_{1} \times G_{2}\right)\right)$ satisfies the Fibered Isomorphism Conjecture if and only if both $\left(G_{1}, \mathcal{C}\left(G_{1}\right)\right)$ and $\left(G_{2}, \mathcal{C}\left(G_{2}\right)\right)$ satisfy the Fibered Isomorphism Conjecture;

(ii) Suppose that $\left(D_{\infty} \times D_{\infty}, \mathcal{V C} y c\left(D_{\infty} \times D_{\infty}\right)\right)$ satisfies the Fibered Isomorphism Conjecture, where $D_{\infty}=\mathbb{Z} \rtimes \mathbb{Z} / 2$ is the infinite dihedral group.

Then $\left(G_{1} \times G_{2}, \mathcal{V C} y c\left(G_{1} \times G_{2}\right)\right)$ satisfies the Fibered Isomorphism Conjecture if and only if both $\left(G_{1}, \mathcal{V C} y c\left(G_{1}\right)\right)$ and $\left(G_{1}, \mathcal{V C} y c\left(G_{1}\right)\right)$ satisfy the Fibered Isomorphism Conjecture.

Proof. (i) If the Fibered Isomorphism Conjecture holds for $\left(G_{1} \times G_{2}, \mathcal{C}\left(G_{1}\right) \times\right.$ $\left.\mathcal{C}\left(G_{2}\right)\right)$, it holds by Lemma 1.5 also for $\left(G_{i}, \mathcal{C}\left(G_{i}\right)\right)$ since $\left.\mathcal{C}\left(G_{1} \times G_{2}\right)\right|_{G_{i}}=\mathcal{C}\left(G_{i}\right)$.

Suppose that the Fibered Isomorphism Conjecture holds for both $\left(G_{1}, \mathcal{C}\left(G_{1}\right)\right)$ and $\left(G_{2}, \mathcal{C}\left(G_{2}\right)\right)$. In view of Lemma 1.9 we can assume without loss of generality that $G_{2}$ belongs to $\mathcal{C}$. Applying this argument again, we can assume without loss of generality that $G_{1}$ and $G_{2}$ belong $\mathcal{C}$. This case is obviously true since $G_{1} \times G_{2} \in \mathcal{C}$. (Compare [58, Lemma 5.1] for a similar argument.)

(ii) Analogously to the proof of assertion[(i), one reduces the claim to the assertion that $\left(G_{1} \times G_{2}, \mathcal{V} \mathcal{C} y c\left(G_{1} \times G_{2}\right)\right)$ satisfy the Fibered Isomorphism Conjecture if $G_{1}$ and $G_{2}$ are virtually cyclic. Since every virtually cyclic group admits an epimorphism to $\mathbb{Z}$ or $D_{\infty}$ with finite kernel and $\mathbb{Z} \subseteq D_{\infty}$, the product $G_{1} \times G_{2}$ admits a group homomorphism to $D_{\infty} \times D_{\infty}$ with finite kernel. Now apply Lemma 1.5 and Lemma 1.10.

Assertion (i) appearing in Lemma 1.11 is interesting in the case $\mathcal{C}=\mathcal{F}$ in.

Lemma 1.12. Suppose that $\mathcal{H}_{*}^{?}$ is continuous. Suppose that any virtually finitely generated abelian group satisfies the Fibered Isomorphism Conjecture for $\mathcal{F}$ in.

Then every elementary amenable group satisfies the Fibered Isomorphism Conjecture for $\mathcal{F}$ in.

Proof. Using the same transfinite induction strategy and the same notation as in the proof of [30, Corollary 3.9], one reduces the claim to the following assertion. The group $G$ satisfies the Fibered Isomorphism Conjecture for $\mathcal{F}$ in provided that there exists an extension $1 \rightarrow H \rightarrow G \stackrel{p}{\rightarrow} A \rightarrow 1$ such that $A$ is virtually finitely generated abelian, $H$ belongs to $L \mathcal{X}_{\alpha-1}$ and the Fibered Isomorphism Conjecture for $\mathcal{F}$ in holds for every group in the class of groups $\mathcal{X}_{\alpha-1}$. Here $L \mathcal{X}_{\alpha-1}$ is the class of groups for which every finitely generated subgroup occurs as a subgroup of some group in $\mathcal{X}_{\alpha-1}$. Theorem 1.7](i) and Lemma 1.5) imply that the Fibered Isomorphism Conjecture holds for every group in $L \mathcal{X}_{\alpha-1}$. Because of Lemma 1.9 it remains to prove for any finite subgroup $K \subseteq A$ that the Fibered Isomorphism Conjecture for $\mathcal{F}$ in holds for $p^{-1}(K)$. We conclude from the short exact sequence $1 \rightarrow H \rightarrow p^{-1}(K) \rightarrow K \rightarrow 1$ and [30, Lemma 2.1 (iii)] that $p^{-1}(K)$ is a member of $L \mathcal{X}_{\alpha-1}$ and satisfies therefore the Fibered Isomorphism Conjecture for $\mathcal{F}$ in. 
Lemma 1.13. Suppose that $\mathcal{H}_{*}^{?}$ is continuous. Suppose that any virtually finitely generated abelian group satisfies the Fibered Isomorphism Conjecture for $\mathcal{V C} y$.

Then every virtually nilpotent group satisfies the Fibered Isomorphism Conjecture for $\mathcal{V C} y c$.

Proof. Any finitely generated subgroup of a virtually abelian group is virtually finitely generated abelian. The assumptions and Theorem 1.7)(i) imply that any virtually abelian group satisfies the Fibered Isomorphism Conjecture for $\mathcal{V C}$ yc.

For a group $H$ we denote by $\operatorname{cent}(H)$ its center. Recall that a group $N$ is called nilpotent, if we can find a sequence of epimorphisms $N=N_{0} \stackrel{p_{0}}{\longrightarrow} N_{1} \stackrel{p_{1}}{\longrightarrow} \cdots \stackrel{p_{r}}{\longrightarrow} N_{r}$ such that $\operatorname{ker}\left(p_{i}\right)=\operatorname{cent}\left(N_{i-1}\right)$ for $i=1,2, \ldots, r$ and $N_{r}=\{1\}$. The class of $N$ is the smallest non-negative integer $r$ for which such a sequence of epimorphisms exists. Let $G$ be virtually nilpotent. Hence we can find a normal subgroup $N \subseteq G$ such that $N$ is nilpotent and $G / N$ is finite. We show by induction over the class of $N$ that $G$ satisfies the Fibered Isomorphism Conjecture for $(G, \mathcal{V C}$ yc). The induction beginning, where the class is $\leq 1$ and hence $G$ is virtually abelian, has already been taken care of.

We can arrange that $r$ is the class of $N$. Since $N$ is normal in $G$ and $\operatorname{cent}(N)$ is a characteristic subgroup of $N$, $\operatorname{cent}(N)$ is a normal subgroup of $G$. We obtain the exact sequence $1 \rightarrow N / \operatorname{cent}(N) \rightarrow G / \operatorname{cent}(N) \rightarrow G / N \rightarrow 1$. Since the class of $N / \operatorname{cent}(N)$ is smaller than the class of $N$, the Fibered Isomorphism Conjecture holds for $(G / \operatorname{cent}(N), \mathcal{V C}$ yc $)$ by the induction hypothesis. Because of Theorem 1.9 it remains to show for any virtually cyclic subgroup $V \subseteq G / \operatorname{cent}(N)$ that $\left(q^{-1}(V), \mathcal{V} \mathcal{C y c}\right)$ satisfies the Fibered Isomorphism Conjecture, where $q: G \rightarrow$ $G / \operatorname{cent}(N)$ is the canonical projection.

Let $\phi: G \rightarrow \operatorname{aut}(N)$ be the group homomorphism sending $g \in G$ to the automorphism of $N$ given by conjugation with $g$. Since $\operatorname{cent}(N)$ is a characteristic subgroup of $N$, it induces a homomorphism $\phi^{\prime}: G \rightarrow \operatorname{aut}(\operatorname{cent}(N))$. Since the conjugation action of $N$ on itself is the identity on $\operatorname{cent}(N)$, the homomorphism $\phi^{\prime}$ factorizes through the finite group $G / N$ and hence has finite image. Hence we can find $g \in G$ such that $q(g)$ generates an infinite cyclic subgroup $C$ in $V$ whose index in $V$ is finite and $\phi^{\prime}(g)=\operatorname{id}_{\operatorname{cent}(N)}$. Hence $q^{-1}(C)$ is isomorphic to $\operatorname{cent}(N) \times C$ and has finite index in $q^{-1}(V)$. Therefore $q^{-1}(V)$ is virtually abelian and $\left(q^{-1}(V), \mathcal{V} \mathcal{C}\right.$ c $)$ satisfies the Fibered Isomorphism Conjecture.

\section{The Farrell-Jones Conjecture for algebraic $K$ - Theory}

Recall that the (Fibered) Farrell-Jones Conjecture for algebraic $K$-theory with coefficients in $R$ for the group $G$ is the (Fibered) Isomorphism Conjecture 1.1 in the special case, where the family $\mathcal{F}$ consists of all virtually cyclic subgroups of $G$ and $\mathcal{H}_{*}^{?}$ is the equivariant homology theory $\mathcal{H}_{*}^{?}\left(-; \mathbf{K}_{R}\right)$ associated to the Groupoidsspectrum given by algebraic $K$-theory and $R$ as coefficient ring (see [45, Section 6]). So the Farrell-Jones Conjecture for algebraic $K$-theory with coefficients in $R$ for 
the group $G$ predicts that the map

$$
H_{n}^{G}\left(E_{\mathcal{V} \mathcal{C y c}_{\mathrm{yc}}}(G), \mathbf{K}_{R}\right) \rightarrow K_{n}(R G)
$$

is bijective for all $n \in \mathbb{Z}$. The original source for (Fibered) Farrell-Jones Conjecture is [28, 1.6 on page 257 and 1.7 on page 262].

Recall that the (Fibered) Farrell-Jones Conjecture for homotopy K-theory with coefficients in $R$ for the group $G$ is the (Fibered) Isomorphism Conjecture1.1 in the special case, where the family $\mathcal{F}$ consists of all finite subgroups of $G$ and $\mathcal{H}_{*}^{\text {? }}$ is the equivariant homology theory $\mathcal{H}_{*}^{?}\left(-; \mathbf{K H}_{R}\right)$ associated to the Groupoids-spectrum given by homotopy $K$-theory and $R$ as coefficient ring (see [5, Section 7]). So the Farrell-Jones Conjecture for homotopy $K$-theory with coefficients in $R$ for the group $G$ predicts that the map

$$
H_{n}^{G}\left(E_{\mathcal{F} \text { in }}(G), \mathbf{K H}_{R}\right) \rightarrow K H_{n}(R G)
$$

is bijective for all $n \in \mathbb{Z}$.

The following theorem follows from the main result of Bartels-Lück-Reich [6] together with [7, Corollary 4.3].

Theorem 2.1. The Fibered Farrell-Jones Conjecture for algebraic K-theory is true for every word-hyperbolic group and every coefficient ring.

Next we extend a result of Quinn [54, Theorem 1.2.2] for virtually abelian groups to virtually nilpotent groups.

Theorem 2.2. The Fibered Farrell-Jones Conjecture for algebraic $K$-theory is true for every virtually nilpotent group and every coefficient ring.

Proof. The Fibered Farrell-Jones Conjecture for algebraic $K$-theory is true for every virtually abelian group and every coefficient ring by a result of Quinn 54, Theorem 1.2.2]. (Quinn deals only with commutative coefficient rings but this assumption is not needed in his argument.) Now apply Lemma 1.13.

For more information about groups satisfying the Farrell-Jones Conjecture for algebraic $K$-theory with coefficients in $\mathbb{Z}$ and the Farrell-Jones Conjecture for homotopy $K$-theory $K H_{*}(R G)$ we refer to [45, Sections 5.2 and 5.3] and [5, Theorem 0.5$]$.

Lemma 2.3. The equivariant homology theories $\mathcal{H}_{*}^{?}\left(-; \mathbf{K}_{R}\right)$ and $\mathcal{H}_{*}^{?}\left(-; \mathbf{K H}_{R}\right)$ are strongly continuous.

Proof. We have to show for every directed systems of groups $\left\{G_{i} \mid i \in I\right\}$ with $G=\operatorname{colim}_{i \in I} G_{i}$ that the canonical maps

$$
\begin{aligned}
\underset{i \in I}{\operatorname{colim}} K_{n}\left(R G_{i}\right) & \rightarrow K_{n}(R G) ; \\
\underset{i \in I}{\operatorname{colim}} K H_{n}\left(R G_{i}\right) & \rightarrow K H_{n}(R G),
\end{aligned}
$$


are bijective for all $n \in \mathbb{Z}$. Obviously $R G$ is the colimit of rings $\operatorname{colim}_{i \in I} R G_{i}$. Now the claim follows for $K_{n}(R G)$ for $n \geq 0$ from [53, (12) on page 20]. Using the Bass-Heller-Swan decomposition one gets the results for $K_{n}(R G)$ and also for the Nil-groups $N^{p} K_{n}(R G)$ defined by Bass [10, XII] for all $n \in \mathbb{Z}$ and $p \geq 1$. The claim for $K H_{n}(R G)$ follows from the spectral sequence [67, Theorem 1.3].

We conclude from Theorem 1.7 and Lemma 2.3 that the (Fibered) Farrell-Jones Conjecture is inherited under directed colimits.

\subsection{Extensions.}

Lemma 2.4. Let $1 \rightarrow K \rightarrow G \rightarrow Q \rightarrow 1$ be an extension of groups. Suppose that $K$ is virtually cyclic and $Q$ satisfies the Fibered Farrell-Jones Conjecture with coefficients in $R$.

Then $G$ satisfies the Fibered Farrell-Jones Conjecture with coefficients in $R$.

Proof. Because of Lemma 1.9 it suffices to prove that $(G ; \mathcal{V C}$ yc $(G))$ satisfies the Fibered Farrell Jones Conjecture in the case that $Q$ is virtually cyclic. Choose an infinite cyclic subgroup $C$ of $Q$. Let $\phi: K \rightarrow K$ be the automorphism given by conjugation with an element in $G$ which is mapped to a generator of $C$ under the epimorphism $G \rightarrow Q$. Then $p^{-1}(C)$ is a subgroup of $G$ which has finite index and is isomorphic to the semi-direct product $K \rtimes_{\phi} C$. Since $K$ is virtually cyclic, its automorphism group has finite order. Hence by replacing $C$ by a subgroup of the order of this automorphism group as index, we can arrange that $p^{-1}(C)$ is a subgroup of finite index in $G$ and $p^{-1}(C) \cong K \times C$. Since $K$ is virtually cyclic, we conclude that $G$ contains a subgroup of finite index which is isomorphic to $\mathbb{Z}^{2}$. In particular $G$ is virtually abelian. Hence $(G, \mathcal{V C}$ yc $(G))$ satisfies the Fibered Farrell-Jones Conjecture by Theorem 2.2 .

Lemma 2.5. Let $G_{1}$ and $G_{2}$ be groups. Then $G_{1} \times G_{2}$ satisfies the Fibered FarrellJones Conjecture with coefficients in $R$ if and only if both $G_{1}$ and $G_{2}$ satisfy the Fibered Farrell-Jones Conjecture with coefficients in $R$.

Proof. Because of Lemma 1.11](ii) it suffices to show that $D_{\infty} \times D_{\infty}$ satisfies the Fibered Farrell-Jones Conjecture. This follows from Theorem 2.2

Next we give the proof of Theorem 0.1 .

Proof. (i) This follows from Theorem 2.1 and Theorem 2.2 .

(ii) This follows from Theorem 2.5.

(iii) This follows from Theorem 1.7[(ii) and Lemma 2.3.

(iv) This follows from Lemma 1.5.

A ring $R$ is called regular coherent if every finitely presented $R$-module possesses a finite-dimensional resolution by finitely generated projective $R$-modules. A ring $R$ is regular if and only if it is regular coherent and Noetherian. A group $G$ is called regular or regular coherent respectively if for any regular ring $R$ the group ring $R G$ is regular respectively regular coherent. Poly-ZZ-groups and free groups 
are regular coherent (see [64, page 247]. For more information about these notions we refer to [64, Theorem 19.1].

Theorem 2.6. Suppose that $Q$ is torsionfree and that the Fibered Farrell-Jones Conjecture holds for $Q$. Suppose that $K$ is a regular coherent group. Suppose that $R$ is regular. Let $1 \rightarrow K \stackrel{i}{\rightarrow} G \stackrel{p}{\rightarrow} Q \rightarrow 1$ be an extension of groups.

Then the assembly map

$$
H_{n}\left(B G ; \mathbf{K}_{R}\right) \stackrel{\cong}{\rightrightarrows} K_{n}(R G)
$$

is an isomorphism for $n \in \mathbb{Z}$.

Proof. Let $\Gamma$ be a torsionfree group. The relative assembly map $H_{n}\left(B \Gamma ; \mathbf{K}_{R}\right) \cong$ $H_{n}^{\Gamma}\left(E_{\mathcal{V C} \text { yc }}(\Gamma) ; K_{R}\right)$ is bijective (see [45, Proposition 2.6 on page 686$]$ ) since $R$ is regular and $\Gamma$ is torsionfree. Hence the Farrell-Jones Conjecture for $\Gamma$ and $R$ boils

down to the claim that the assembly map $H_{n}\left(B \Gamma ; \mathbf{K}_{R}\right) \stackrel{\cong}{\rightrightarrows} K_{n}(R \Gamma)$ is bijective for all $n \in \mathbb{Z}$. This implies that Theorem 2.6 follows directly from Lemma 1.8 if we can show that the second assumption appearing in Lemma 1.8 is satisfied.

Let $\mathcal{C L}$ be the class of groups introduced in [64, Definion 19.2 on page 248] or [5. Definition 0.10]. Let $V \subseteq Q$ be virtually cyclic. Since $K$ is regular coherent and $Q$ is torsionfree and hence $V$ is isomorphic to $\mathbb{Z}, p^{-1}(V)$ belongs to the class $\mathcal{C L}$. Since $\mathcal{C L}$ by [64, Proposition 19.3 on page 249] is closed under taking subgroups, every element in $p^{*} \mathcal{V C}$ yc $(Q)$ belongs to $\mathcal{C L}$. One of the main results in Waldhausen's article 64] is that for a regular ring $R$ the $K$-theoretic assembly map $H_{n}\left(B G^{\prime} ; \mathbf{K}_{R}\right) \rightarrow K_{n}\left(R G^{\prime}\right)$ is an isomorphism for $G^{\prime} \in \mathcal{C L}$. Hence the second assumption appearing in Lemma 1.8 is satisfied.

\subsection{Passage from $\mathcal{F}$ in to $\mathcal{V} \mathcal{C}$ yc.}

Lemma 2.7. Let $G$ be a group. Let $R$ be a regular ring such that the order of any finite subgroup of $G$ is invertible in $R$.

Then the relative assembly map

$$
H_{n}^{G}\left(E_{\mathcal{F} i n}(G) ; \mathbf{K}_{R}\right) \rightarrow H_{n}^{G}\left(E_{\mathcal{V} C y c}(G) ; \mathbf{K}_{R}\right)
$$

(induced by the up to $G$-homotopy unique $G$-map $E_{\mathcal{F} \text { in }}(G) \rightarrow E_{\mathcal{V C} y c}(G)$ ) is an isomorphism for all $n \in \mathbb{Z}$.

Proof. This is proved for instance in [45, Proposition 2.6 on page 686].

Lemma 2.8. Let $G$ be a group. Then the relative assembly map induces for all $n \in \mathbb{Z}$ isomorphisms

$$
H_{n}^{G}\left(E_{\mathcal{F} i n}(G) ; \mathbf{K}_{\mathbb{Z}}\right) \otimes_{\mathbb{Z}} \mathbb{Q} \rightarrow H_{n}^{G}\left(E_{\mathcal{V} C y c}(G) ; \mathbf{K}_{\mathbb{Z}}\right) \otimes_{\mathbb{Z}} \mathbb{Q} .
$$

Proof. This is proved in [34, Theorem 5.6]. 
Notice that the two Lemmas 2.7 and 2.8 above deal not with the Fibered version. A discussion of the Fibered version of Lemma 2.7 can be found in Subsection 6.3. The fibered version of Lemma 2.8 is definitely false. For homotopy $K$-theory one can prove in the fibered situation that the passage from $\mathcal{F}$ in to $\mathcal{V C}$ yc does not matter. For $\Lambda=\mathbb{Z}$ the next lemma is proven in [5, Remark 7.4]. The following more general statement follows by the same argument.

Lemma 2.9. Let $R$ be a ring. A group $G$ satisfies the (Fibered) Isomorphism Conjecture for homotopy $K$-theory with coefficients in $R$ for the family $\mathcal{F}$ in after applying $-\otimes_{\mathbb{Z}} \Lambda$ for $\mathbb{Z} \subseteq \Lambda \subseteq \mathbb{Q}$ to the assembly map if and only if $G$ satisfies the (Fibered) Isomorphism Conjecture for homotopy $K$-theory with coefficients in $R$ for the family $\mathcal{V C}$ yc after applying $-\otimes_{\mathbb{Z}} \Lambda$ for $\mathbb{Z} \subseteq \Lambda \subseteq \mathbb{Q}$ to the assembly map.

\subsection{Homotopy $K$-theory and rings with finite characteristic.}

Lemma 2.10. Let $R$ be a ring of finite characteristic $N$. Let $N K_{n}(R)$ be the Nil-group of Bass. Then we get for $n \in \mathbb{Z}$

$$
N K_{n}(R)[1 / N]=0 .
$$

Proof. The proof can be found in [66, Corollary 3.2]. We give a brief outline for the reader's convenience.

Put $\Lambda=\mathbb{Z} / N$. Then $R$ is a $\Lambda$-algebra. Let $W(\Lambda)$ be the ring of big Witt vectors over $\Lambda$. The underlying additive group is the multiplicative group $1+t \Lambda[[t]]$ of formal power series with leading term 1 . We do not need the explicit multiplicative structure but need to know that the identity element is $1-t$. Let $\operatorname{End}(\Lambda)$ be the Grothendieck group of endomorphisms $f: P \rightarrow P$ of finitely generated projective $\Lambda$-modules. We get an injective homomorphism $K_{0}(\Lambda) \rightarrow \operatorname{End}(\Lambda)$ by sending $[P]$ to $[0: P \rightarrow P]$. Its cokernel is denoted by $\operatorname{End}_{0}(\Lambda)$. The tensor product induces the structure of a commutative ring on $\operatorname{End}(\Lambda)$ for which $K_{0}(\Lambda)$ becomes an ideal. Hence $\operatorname{End}_{0}(\Lambda)$ is a commutative ring. There is a $\operatorname{End}_{0}(\Lambda)$-module structure on $N K_{n}(R)$ for all $n \in \mathbb{Z}$. Almkvist [1] shows that the characteristic polynomial defines an injective ring homomorphism

$$
\chi: \operatorname{End}_{0}(\Lambda) \rightarrow W(\Lambda), \quad[f: P \rightarrow P] \mapsto \operatorname{det}\left(\operatorname{id}_{P}-t \cdot f\right) .
$$

For a positive integer $N$ let $I_{N}$ be the ideal in $\operatorname{End}_{0}(\Lambda)$

$$
I_{N}=\left\{[f: P \rightarrow P] \mid \chi([f]) \equiv 1 \bmod t^{N}\right\} .
$$

Stienstra 62] (see also Theorem 1.3 and the following paragraph in [66]) has proven that for every element $x \in N K_{n}(R)$ there exists $N(x)$ such that the ideal $I_{N(x)}$

annihilates $x$. Now choose a sufficiently large positive integer $k$ such that $\left(\begin{array}{c}N^{k} \\ j\end{array}\right)$ is a multiple of $N$ for $1 \leq j<N(x)$. Then we conclude

$$
\chi\left(N^{k} \cdot\left[\operatorname{id}_{\Lambda}\right]\right)=(1-t)^{N^{k}}=\sum_{j=0}^{N^{k}}\left(\begin{array}{c}
N^{k} \\
j
\end{array}\right)(-t)^{j}=1+\sum_{j=N(x)}^{N^{k}}\left(\begin{array}{c}
N^{k} \\
j
\end{array}\right)(-t)^{j} \equiv 1 \quad \bmod t^{N(x)} .
$$


This shows that $N^{k} \cdot\left[\operatorname{id}_{\Lambda}\right] \in I_{N(x)}$. We compute

$$
N^{k} \cdot x=N^{k} \cdot\left(\left[\operatorname{id}_{\Lambda}\right] \cdot x\right)=\left(N^{k} \cdot\left[\operatorname{id}_{\Lambda}\right]\right) \cdot x=0 .
$$

This implies $N K_{n}(R)[1 / N]=0$.

Lemma 2.11. (i) Let $R$ be a ring of finite characteristic $N$. Then the canonical map from algebraic $K$-theory to homotopy $K$-theory induces an isomorphism

$$
K_{n}(R)[1 / N] \stackrel{\cong}{\rightarrow} K H_{n}(R)[1 / N]
$$

for all $n \in \mathbb{Z}$

(ii) Let $H$ be a finite group. Then the canonical map from algebraic $K$-theory to homotopy $K$-theory induces an isomorphism

$$
K_{n}(\mathbb{Z}[H]) \otimes_{\mathbb{Z}} \mathbb{Q} \cong K H_{n}(\mathbb{Z}[H]) \otimes_{\mathbb{Z}} \mathbb{Q}
$$

for all $n \in \mathbb{Z}$.

Proof. (i) We conclude from Lemma 2.10 that $N K_{n}(R)[1 / N]=0$. This implies that $N^{p} K_{n}(R)[1 / N]=0$ for $n \in \mathbb{Z}$ and $p \geq 1$. Now apply the spectral sequence from [67, Theorem 1.3].

(ii) By [66, Corollary] and [5, Remark 8.3] $N K_{n}(\mathbb{Z}[H]) \otimes_{\mathbb{Z}} \mathbb{Q}=0$. Now proceed as in (i)

Lemma 2.12. Let $(X, A)$ be a pair of $G$-CW-complexes.

(i) Let $R$ be a ring of finite characteristic $N$. Then the natural map

$$
H_{n}^{G}\left(X, A ; \mathbf{K}_{R}\right)[1 / N] \rightarrow H_{n}^{G}\left(X, A ; \mathbf{K H}_{R}\right)[1 / N]
$$

is bijective for every $n \in \mathbb{Z}$;

(ii) Assume that $X$ is a $G$-Fin-CW-complex, i.e., the isotropy groups of $X$ are finite. Then the natural map

$$
H_{n}^{G}\left(X, A ; \mathbf{K}_{\mathbb{Z}}\right) \otimes_{\mathbb{Z}} \mathbb{Q} \rightarrow H_{n}^{G}\left(X, A ; \mathbf{K H}_{\mathbb{Z}}\right) \otimes_{\mathbb{Z}} \mathbb{Q}
$$

is bijective for every $n \in \mathbb{Z}$.

Proof. This follows from Lemma 2.11 and a spectral sequence argument based on the equivariant Atiyah-Hirzebruch spectral sequence (see for instance [20, Theorem 4.7]).

Lemma 2.13. Let $R$ be a ring and let $G$ be a group. Let $N \geq 2$ be an integer.

(i) If $G$ satisfies the (Fibered) Farrell-Jones Conjecture for algebraic $K$-theory with coefficients in $R\left[x_{1}, x_{2}, \ldots, x_{k}\right]$ for all $k \geq 0$, then $G$ satisfies the (Fibered) Farrell-Jones Conjecture for homotopy $K$-theory with coefficients in $R$; 
(ii) Suppose that $N \cdot 1_{R}=0$ and that $G$ satisfies the (Fibered) Farrell-Jones Conjecture for homotopy $K$-theory with coefficients in $R$ after applying $-\otimes_{\mathbb{Z}}$ $\mathbb{Z}[1 / N]$. Then $G$ satisfies the (Fibered) Farrell-Jones Conjecture for algebraic $K$-theory with coefficients in $R$ after applying $-\otimes_{\mathbb{Z}} \mathbb{Z}[1 / N]$ for both the family $\mathcal{F}$ in and $\mathcal{V C} y c$.

Proof. (i) This is proven in [5, Theorem 8.4].

(ii) Consider for any family $\mathcal{F}$ of subgroups of $G$ the following commutative diagram

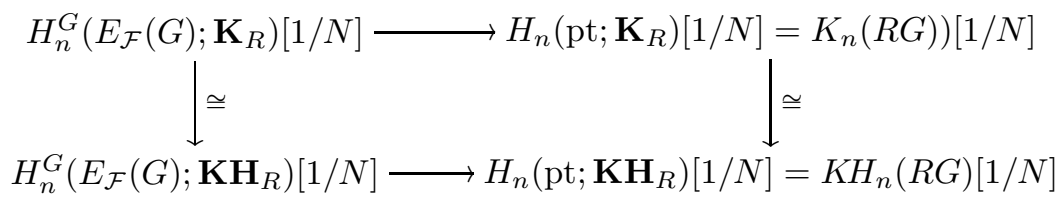

where the horizontal maps are the assembly maps induced by the projection $E_{\mathcal{F}}(G) \rightarrow$ pt and the vertical maps are induced by the passage from algebraic $K$-theory to homotopy $K$-theory. Lemma 2.12 (i) implies that the vertical maps are bijective. Now apply Lemma 2.9.

Next we can give the proof of Theorem 0.3 .

Proof. [i) Word-hyperbolic groups and virtually abelian groups satisfy the FarrellJones Conjecture for algebraic $K$-theory with coefficients in any ring $R$ by Theorem 2.1 and Theorem 2.2. We conclude from Lemma 2.13 (i) that word-hyperbolic groups and virtually abelian groups belong to $\mathcal{F J}_{K H}(R)$ for all rings $R$. We conclude from Lemma 1.12 that all elementary amenable groups belong to $\mathcal{F J}_{K H}(R)$ for all rings $R$. Lemma 2.13 (ii) implies that all word-hyperbolic groups and all elementary amenable groups belong to $\mathcal{F J}_{F C}(R)$.

In particular every virtually cyclic group belongs to $\mathcal{F J}_{F C}(R)$. We conclude from Theorem 1.4 that for a ring $R$ of characteristic $N$ a group satisfies the Fibered Farrell-Jones Conjecture for algebraic $K$-theory for $G$ with coefficients in $R$ after applying $-\otimes_{\mathbb{Z}} \mathbb{Z}[1 / N]$ to the assembly map for the family $\mathcal{F}$ in if and only it does for the family $\mathcal{V} \mathcal{C}$ yc. Hence in the sequel we only have to consider the family $\mathcal{F}$ in when dealing with $\mathcal{F J}_{F C}(R)$.

(ii) This follows from Lemma 1.11 (i)

(iii) This follows from Theorem 1.7[(ii) and Lemma 2.3.

(iv) This follows from Lemma 1.5 .

(v) This follows from Lemma 1.9 since a group which is commensurable to a wordhyperbolic group is again word-hyperbolic and the same is true for elementary amenable groups.

(vi) This is proven for $\mathcal{F J}_{K H}$ in Bartels-Lück [4, Theorem 0.5]. The same proof applies to $\mathcal{F J}_{F C}$ if we can show that $\mathcal{H}_{*}^{\text {? }}\left(-; \mathbf{K}_{R}\right)[1 / N]$ has the tree property (see [4, Definition 4.1 and Theorem 4.2]). This follows from Lemma2.12 (i)] since the equivariant homology theory $\mathcal{H}_{*}^{?}\left(-; \mathbf{K H}_{R}\right)$ has the tree property by [4, Theorem 11.1].

The claim $\mathcal{F J}_{K H}(R) \subseteq \mathcal{F}_{F C}(R)$ follows from Lemma 2.13 (ii). 
2.4. Coefficient rings with operation. In the setup developed so far we have not dealt with the more general version developed in Bartels-Reich [7] where one fixes a group and considers an additive category with $G$-action. This setup can deal with crossed products $R \rtimes G$ and not only with group rings $R G$. However, a slight modification of the proofs above allows to carry over the result above to this setting. This is explained in Bartels-Echterhoff-Lück [3].

\section{The projective class group and induction from finite subgroups}

Let $\operatorname{Or}_{\mathcal{F} \text { in }}(G)$ be the category whose objects are homogeneous spaces $G / H$ with finite $H$ and whose morphisms are $G$-maps. We obtain a functor from $\operatorname{Or}_{\mathcal{F i n}}(G)$ to abelian groups by sending $G / H$ to $K_{0}(R H)$. It sends a morphism $G / H \rightarrow$ $G / K, g H \mapsto g g_{0} K$ to the map $K_{0}(R H) \rightarrow K_{0}(R K)$ coming from the group homomorphism $H \rightarrow K, h \mapsto g_{0} h g_{0}^{-1}$. This is well-defined since inner automorphisms of $H$ induce the identity on $K_{0}(R H)$. The various inclusions of finite subgroups of $G$ yield a homomorphism

$$
I(G ; R): \underset{\operatorname{Or}_{\mathcal{F i n}}(G)}{\operatorname{colim}} K_{0}(R H) \rightarrow K_{0}(R G) .
$$

Notice for the sequel that the canonical map of $\Lambda$-modules

$$
\left(\underset{\operatorname{Or}_{\mathcal{F i n}}(G)}{\operatorname{colim}} K_{0}(R H)\right) \otimes_{\mathbb{Z}} \Lambda \stackrel{\cong}{\rightarrow} \underset{\operatorname{Or}_{\mathcal{F i n}}(G)}{\operatorname{colim}}\left(K_{0}(R H) \otimes_{\mathbb{Z}} \Lambda\right)
$$

is bijective for every ring $\Lambda$.

The next lemma is proven in [30, Lemma 2.9] for fields and carries over directly to skew-fields.

Lemma 3.1. Let $D$ be a skew-field (of arbitrary characteristic) and $H$ be a finite group. Then $K_{n}(D H)=0$ for $n \leq-1$.

Now we can give the proof of Theorem 0.5

Proof. (i) This is proved in [45, page 691].

(ii) This follows analogously to the proof of assertion (i) using Lemma 2.10 and Lemma 3.1 .

If $G$ satisfies the Farrell-Jones Conjecture for algebraic $K$-theory with coefficients in $\mathbb{Z}$, then the following maps are injective (see [45, page 692]).

$$
\begin{aligned}
\underset{\operatorname{Or}_{\mathcal{F i n}}(G)}{\operatorname{colim}} K_{n}(\mathbb{Z} H) \otimes_{\mathbb{Z}} \mathbb{Q} & \rightarrow K_{n}(\mathbb{Z} G) \otimes_{\mathbb{Z}} \mathbb{Q} \quad \text { for } n \in \mathbb{Z} ; \\
\underset{\operatorname{Or}_{\mathcal{F i n}}(G)}{\operatorname{colim}} \operatorname{Wh}(H) \otimes_{\mathbb{Z}} \mathbb{Q} & \rightarrow \mathrm{Wh}(G) \otimes_{\mathbb{Z}} \mathbb{Q} .
\end{aligned}
$$


The injectivity for the map involving the Whitehead group is proven for groups $G$ satisfying a mild homological finiteness conditions in [47. In general these maps are not surjective. In particular

$$
\underset{\operatorname{Or}_{\mathcal{F} \text { in }}(G)}{\operatorname{colim}} K_{0}(\mathbb{Z} H) \otimes_{\mathbb{Z}} \mathbb{Q} \rightarrow K_{n}(\mathbb{Z} G) \otimes_{\mathbb{Z}} \mathbb{Q}
$$

is in general not surjective.

\section{Bass Conjectures}

In this section we explain the relationship between the Farrell-Jones Conjecture for algebraic $K$-theory and the Bass Conjecture.

Let $G$ be a group. Let $\operatorname{con}(G)$ be the set of conjugacy classes $(g)$ of elements $g \in G$. Denote by $\operatorname{con}(G)_{f}$ the subset of $\operatorname{con}(G)$ consisting of those conjugacy classes $(g)$ for which each representative $g$ has finite order. Let $R$ be a commutative ring. Let $\operatorname{class}_{R}(G)$ and $\operatorname{class}_{R}(G)_{f}$ be the free $R$-module with the set $\operatorname{con}(G)$ and $\operatorname{con}(G)_{f}$ as basis. This is the same as the $R$-module of $R$-valued functions on $\operatorname{con}(G)$ and $\operatorname{con}(G)_{f}$ with finite support. Define the universal $R$-trace

$$
\operatorname{tr}_{R G}^{u}: R G \rightarrow \operatorname{class}_{R}(G), \quad \sum_{g \in G} r_{g} \cdot g \mapsto \sum_{g \in G} r_{g} \cdot(g) .
$$

It extends to a function $\operatorname{tr}_{R G}^{u}: M_{n}(R G) \rightarrow \operatorname{class}_{R}(G)$ on $(n, n)$-matrices over $R G$ by taking the sum of the traces of the diagonal entries. Let $P$ be a finitely generated projective $R G$-module. Choose a matrix $A \in M_{n}(R G)$ such that $A^{2}=A$ and the image of the $R G$-map $r_{A}: R G^{n} \rightarrow R G^{n}$ given by right multiplication with $A$ is $R G$-isomorphic to $P$. Define the Hattori-Stallings rank of $P$ as

$$
\operatorname{HS}_{R G}(P)=\operatorname{tr}_{R G}^{u}(A) \quad \in \operatorname{class}_{R}(G) .
$$

The Hattori-Stallings rank depends only on the isomorphism class of the $R G$ module $P$. It induces an $R$-homomorphism, the Hattori-Stallings homomorphism,

$$
\mathrm{HS}_{R G}: K_{0}(R G) \otimes_{\mathbb{Z}} R \rightarrow \operatorname{class}_{R}(G), \quad[P] \otimes r \mapsto r \cdot \operatorname{HS}_{R G}(P) .
$$

Let $F$ be a field of characteristic zero. Fix an integer $m \geq 1$. Let $F\left(\zeta_{m}\right) \supset F$ be the Galois extension given by adjoining the primitive $m$-th root of unity $\zeta_{m}$ to $F$. Denote by $\Gamma(m, F)$ the Galois group of this extension of fields, i.e., the group of automorphisms $\sigma: F\left(\zeta_{m}\right) \rightarrow F\left(\zeta_{m}\right)$ which induce the identity on $F$. It can be identified with a subgroup of $\mathbb{Z} / m^{*}$ by sending $\sigma$ to the unique element $u(\sigma) \in \mathbb{Z} / m^{*}$ for which $\sigma\left(\zeta_{m}\right)=\zeta_{m}^{u(\sigma)}$ holds. Let $g_{1}$ and $g_{2}$ be two elements of $G$ of finite order. We call them $F$-conjugate if for some (and hence all) positive integers $m$ with $g_{1}^{m}=g_{2}^{m}=1$ there exists an element $\sigma$ in the Galois group $\Gamma(m, F)$ with the property that $g_{1}^{u(\sigma)}$ and $g_{2}$ are conjugate. Two elements $g_{1}$ and $g_{2}$ are $F$-conjugate for $F=\mathbb{Q}, \mathbb{R}$ or $\mathbb{C}$ respectively if the cyclic subgroups $\left\langle g_{1}\right\rangle$ and 
$\left\langle g_{2}\right\rangle$ are conjugate, if $g_{1}$ and $g_{2}$ or $g_{1}$ and $g_{2}^{-1}$ are conjugate, or if $g_{1}$ and $g_{2}$ are conjugate respectively.

Denote by $\operatorname{con}_{F}(G)_{f}$ the set of $F$-conjugacy classes $(g)_{F}$ of elements $g \in G$ of finite order. Let $\operatorname{class}_{F}(G)_{f}$ be the $F$-vector space with the set $\operatorname{con}_{F}(G)_{f}$ as basis, or, equivalently, the $F$-vector space of functions $\operatorname{con}_{F}(G)_{f} \rightarrow F$ with finite support. There are obvious inclusions of $F$-modules $\operatorname{class}_{F}(G)_{f} \subseteq \operatorname{class}_{F}(G)$.

Lemma 4.1. Suppose that $F$ is a field of characteristic zero and $H$ is a finite group. Then the Hattori-Stallings homomorphism (see (4.3) induces an isomorphism

$$
\mathrm{HS}_{F H}: K_{0}(F H) \otimes_{\mathbb{Z}} F \stackrel{\cong}{\operatorname{class}_{F}}(H)=\operatorname{class}_{F}(H)_{f}
$$

Proof. Since $H$ is finite, an $F H$-module is a finitely generated projective $F H$ module if and only if it is a (finite-dimensional) $H$-representation with coefficients in $F$ and $K_{0}(F H)$ is the same as the representation $\operatorname{ring} R_{F}(H)$. The HattoriStallings rank $\operatorname{HS}_{F H}(V)$ and the character $\chi_{V}$ of a $G$-representation $V$ with coefficients in $F$ are related by the formula

$$
\chi_{V}(h)=\left|Z_{G}\langle h\rangle\right| \cdot \operatorname{HS}_{F H}(V)(h)
$$

for $h \in H$, where $Z_{G}\langle h\rangle$ is the centralizer of $h$ in $G$. Hence Lemma 4.1 follows from representation theory, see for instance [60, Corollary 1 on page 96].

Notice that the Bass Conjecture for fields of characteristic zero as coefficients 0.8 is the obvious generalization of Lemma 4.1 to infinite groups.

Lemma 4.2. Suppose that $F$ is a field of characteristic zero and $G$ is a group. Then the composite

$$
\underset{G / H \in \operatorname{Or}_{\mathcal{F}_{i n}}(G)}{\operatorname{colim}} K_{0}(F H) \otimes_{\mathbb{Z}} F \stackrel{I(G, F) \otimes_{\mathbb{Z}} \operatorname{id}_{F}}{\longrightarrow} K_{0}(F G) \otimes_{\mathbb{Z}} F \stackrel{\operatorname{HS}_{F G}}{\longrightarrow} \operatorname{class}_{F}(G)
$$

is injective and has as image $\operatorname{class}_{F}(G)_{f}$.

Proof. This follows from the following commutative diagram, compare 39, Lemma 2.15 on page 220].

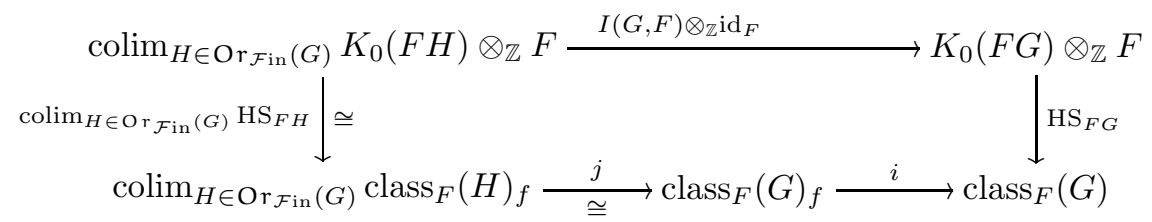

Here the isomorphism $j$ is the colimit over the obvious maps $\operatorname{class}_{F}(H)_{f} \rightarrow$ $\operatorname{class}_{F}(G)_{f}$ given by extending a class function in the trivial way and the map $i$ is the natural inclusion and in particular injective. 
Lemma 4.3. Let $F$ be a field (of arbitrary characteristic). Then there is a natural commutative diagram

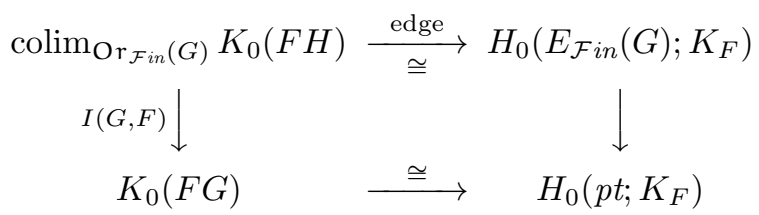

whose horizontal maps are bijective and whose right vertical arrow is the assembly map, i.e., the map induced by the projection $E_{\mathcal{F} \text { in }}(G) \rightarrow p t$.

Proof. This follows from Lemma 3.1, the equivariant Atiyah-Hirzebruch spectral sequence, which converges to $H_{p+q}\left(E_{\mathcal{F} \text { in }}(G) ; K_{F}\right)$ in the strong sense and whose $E_{2}$-term $E_{p, q}^{2}$ is given by the Bredon homology $H_{p}^{\mathrm{Or} \operatorname{F}_{\mathcal{F i n}}(G)}\left(E_{\mathcal{F} \text { in }}(G), K_{q}(F ?)\right)$, and the natural identification

$$
\operatorname{colim}_{\operatorname{Or}_{\mathcal{F i n}}(G)} K_{0}(F H) \cong H_{0}^{\mathrm{Or}_{\mathcal{F} \text { in }}(G)}\left(E_{\mathcal{F} \text { in }}(G), K_{0}(F ?)\right)
$$

Theorem 0.9 follows now from Lemma 2.7. Lemma 4.2 and Lemma 4.3 .

Lemma 4.4. Let $R$ be a commutative integral domain. Let $F$ be its quotient field. Let $G$ be a group.

(i) Then we obtain a commutative diagram

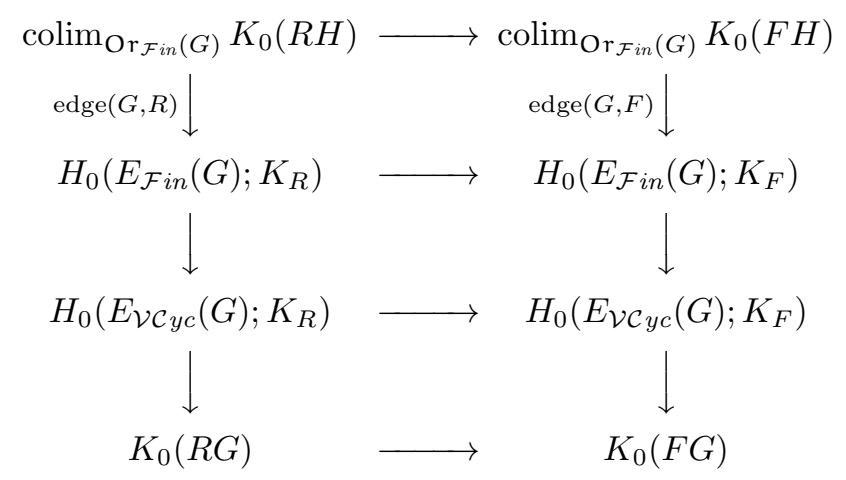

where all horizontal maps are change of rings homomorphisms for the inclusion $R \rightarrow F$, the maps edge $(G, R)$ and $\operatorname{edge}(G, F)$ are edge homomorphisms appearing in the equivariant Atiyah-Hirzebruch spectral sequence and the other vertical maps come from the obvious maps $E_{\mathcal{F} \text { in }}(G) \rightarrow E_{\mathcal{V C} y c}(G)$ and $E_{\mathcal{V} C y c}(G) \rightarrow p t$

(ii) The image of the composite

$$
\alpha: H_{0}\left(E_{\mathcal{V} C y c}(G) ; K_{R}\right) \otimes_{\mathbb{Z}} \mathbb{Q} \rightarrow H_{0}\left(E_{\mathcal{V} C y c}(G) ; K_{F}\right) \otimes_{\mathbb{Z}} \mathbb{Q} \rightarrow K_{0}(F G) \otimes_{\mathbb{Z}} \mathbb{Q}
$$


is the same as the image of the composite

$$
\beta: \bigoplus_{C \in \mathcal{F C} y c(G)} K_{0}(R C) \otimes_{\mathbb{Z}} \mathbb{Q} \rightarrow \bigoplus_{C \in \mathcal{F} \mathcal{C} y c(G)} K_{0}(F C) \otimes_{\mathbb{Z}} \mathbb{Q} \rightarrow K_{0}(F G) \otimes_{\mathbb{Z}} \mathbb{Q}
$$

where $\mathcal{F C}$ yc is the family all finite cyclic subgroups of $G$.

Proof. (i) This follows from the naturality of the constructions.

(ii) For every group $G$, every $\operatorname{ring} R$, and every $n \in \mathbb{Z}$ the relative assembly map

$$
H_{n}^{G}\left(E_{\mathcal{F} \text { in }}(G) ; \mathbf{K}_{R}\right) \rightarrow H_{n}^{G}\left(E_{\mathcal{V} \mathcal{C}_{\text {yc }}}(G) ; \mathbf{K}_{R}\right)
$$

is split-injective [9]. This map and the splitting are natural with respect to change of rings homomorphisms. Hence Lemma 2.7 implies that the image of

$$
H_{n}^{G}\left(E_{\mathcal{V} \mathcal{C y c}_{\mathrm{yc}}}(G) ; \mathbf{K}_{R}\right) \otimes_{\mathbb{Z}} \mathbb{Q} \rightarrow H_{n}^{G}\left(E_{\mathcal{V} \mathcal{C y c}}(G) ; \mathbf{K}_{F}\right) \otimes_{\mathbb{Z}} \mathbb{Q}
$$

and the image of the composite

$$
H_{n}^{G}\left(E_{\mathcal{F} \text { in }}(G) ; \mathbf{K}_{R}\right) \otimes_{\mathbb{Z}} \mathbb{Q} \rightarrow H_{n}^{G}\left(E_{\mathcal{F} \text { in }}(G) ; \mathbf{K}_{F}\right) \otimes_{\mathbb{Z}} \mathbb{Q} \rightarrow H_{n}^{G}\left(E_{\mathcal{V} \mathcal{C}_{\text {yc }}}(G) ; \mathbf{K}_{F}\right) \otimes_{\mathbb{Z}} \mathbb{Q}
$$

agree. By Theorem 1.13 in [46], a variation of the Chern character in [40], we have for every $\operatorname{ring} R$ and group $G$ an isomorphism, natural in $R$,

$$
\begin{aligned}
\operatorname{chern}_{n}^{G, R}: \bigoplus_{p+q=n} \bigoplus_{\substack{C \in \mathcal{F} \mathcal{C} \text { yc }(G)\\
}} H_{p}\left(B Z_{G} C ; \mathbb{Q}\right) & \otimes_{\mathbb{Q}\left[W_{G} C\right]} \theta_{C} \cdot\left(K_{q}(R C) \otimes_{\mathbb{Z}} \mathbb{Q}\right) \\
& \rightarrow H_{n}^{G}\left(E_{\mathcal{F} \text { in }}(G) ; K_{R}\right) \otimes_{\mathbb{Z}} \mathbb{Q}
\end{aligned}
$$

where $W_{G} C$ is the quotient of the normalizer by the centralizer $Z_{G} C$ of the finite cyclic subgroup $C$ in $G$ and $\theta_{C}$ is an idempotent in $A(C) \otimes_{\mathbb{Z}} \mathbb{Q}$ for $A(C)$ the Burnside ring of $C$ which singles out a direct summand $\theta_{C} \cdot\left(K_{q}(R C) \otimes_{\mathbb{Z}} \mathbb{Q}\right)$ in $K_{q}(R C) \otimes_{\mathbb{Z}} \mathbb{Q}$ by the $A(C) \otimes_{\mathbb{Z}} \mathbb{Q}$-module structure on $K_{q}(R C) \otimes_{\mathbb{Z}} \mathbb{Q}$. This isomorphism is natural under change of rings homomorphisms. Recall from Lemma 3.1 that $K_{q}(F C)$ is trivial for $q \leq-1$. Hence the image of

$$
H_{0}^{G}\left(E_{\mathcal{F} \text { in }}(G) ; \mathbf{K}_{R}\right) \otimes_{\mathbb{Z}} \mathbb{Q} \rightarrow H_{0}^{G}\left(E_{\mathcal{F} \text { in }}(G) ; \mathbf{K}_{F}\right) \otimes_{\mathbb{Z}} \mathbb{Q}
$$

agrees with the image of the composition

$$
\begin{gathered}
\bigoplus_{(C) \in \mathcal{F C y c}(G)} H_{0}\left(B Z_{G} C ; \mathbb{Q}\right) \otimes_{\mathbb{Q}\left[W_{G} C\right]} \theta_{C} \cdot\left(K_{0}(R C) \otimes_{\mathbb{Z}} \mathbb{Q}\right) \rightarrow \\
\bigoplus_{(C) \in \mathcal{F} \mathcal{C y c}(G)} H_{0}\left(B Z_{G} C ; \mathbb{Q}\right) \otimes_{\mathbb{Q}\left[W_{G} C\right]} \theta_{C} \cdot\left(K_{0}(F C) \otimes_{\mathbb{Z}} \mathbb{Q}\right) \\
=\bigoplus_{p+q=0} \bigoplus_{(C) \in \mathcal{F} C \mathrm{yc}(G)} H_{p}\left(B Z_{G} C ; \mathbb{Q}\right) \otimes_{\mathbb{Q}\left[W_{G} C\right]} \theta_{C} \cdot\left(K_{q}(F C) \otimes_{\mathbb{Z}} \mathbb{Q}\right) \\
\stackrel{\operatorname{chern}_{0}^{G, F}}{\longrightarrow} H_{0}^{G}\left(E_{\mathcal{F} \text { in }}(G) ; K_{F}\right) \otimes_{\mathbb{Z}} \mathbb{Q} .
\end{gathered}
$$


Since $H_{0}\left(B Z_{G} C ; \mathbb{Q}\right)$ is $\mathbb{Q}$ with the trivial $W_{G} C$-action, $H_{0}\left(B Z_{G} C ; \mathbb{Q}\right) \otimes_{\mathbb{Q}\left[W_{G} C\right]}$ $\theta_{C} \cdot\left(K_{0}(R C) \otimes_{\mathbb{Z}} \mathbb{Q}\right)$ is a quotient of $\theta_{C} \cdot\left(K_{0}(R C) \otimes_{\mathbb{Z}} \mathbb{Q}\right)$ which is a direct summand in $K_{0}(R C) \otimes_{\mathbb{Z}} \mathbb{Q}$. Hence $\operatorname{im}(\alpha) \subseteq \operatorname{im}(\beta)$. We get $\operatorname{im}(\beta) \subseteq \operatorname{im}(\alpha)$ from the commutative diagram appearing in assertion (i). This finishes the proof of Lemma 4.4

Finally we can give the proof of Theorem 0.10

Proof. For any finite group of $H \subseteq G$ such that its order is not invertible in $R$ the map $\widetilde{K}_{0}(R H) \rightarrow \widetilde{K}_{0}(F H)$ is trivial by a result of Swan [63, Theorem 8.1] (see also [11, Corollary 4.2]). Lemma 4.4](ii) implies that the composite

$H_{0}\left(E_{\mathcal{V} \mathcal{C y c}_{\mathrm{c}}}(G) ; K_{R}\right) \otimes_{\mathbb{Z}} \mathbb{Q} \rightarrow H_{0}\left(E_{\mathcal{V} \mathcal{C}_{\mathrm{yc}}}(G) ; K_{F}\right) \otimes_{\mathbb{Z}} \mathbb{Q} \rightarrow K_{0}(F G) \otimes_{\mathbb{Z}} \mathbb{Q} \rightarrow \widetilde{K}_{0}(F G) \otimes_{\mathbb{Z}} \mathbb{Q}$

is trivial. Since $G$ satisfies the Farrell-Jones Conjecture for algebraic $K$-theory with coefficients in $R$ by assumption, the map $H_{0}\left(E_{\mathcal{V} \mathcal{C}_{\text {yc }}}(G) ; K_{R}\right) \otimes_{\mathbb{Z}} \mathbb{Q} \rightarrow K_{0}(R G) \otimes_{\mathbb{Z}} \mathbb{Q}$ is surjective. Because of the commutative diagram appearing in Lemma 4.4 (i) the map

$$
K_{0}(R G) \otimes_{\mathbb{Z}} \mathbb{Q} \rightarrow K_{0}(F G) \otimes_{\mathbb{Z}} \mathbb{Q} \rightarrow \widetilde{K}_{0}(F G) \otimes_{\mathbb{Z}} \mathbb{Q}
$$

is trivial. Now Theorem 0.10 follows.

\section{The Kaplansky Conjecture}

We need the following definition.

Definition 5.1. An $R$-module $M$ is called directly finite if every $R$-module $N$ satisfying $M \cong_{R} M \oplus N$ is trivial. A ring $R$ is called directly finite (or von Neumann finite) if it is directly finite as a module over itself, i.e., if $r, s \in R$ satisfy $r s=1$, then $s r=1$. A ring is called stably finite if the matrix algebra $M(n, n, R)$ is directly finite for all $n \geq 1$.

Remark 5.2. Stable finiteness for a ring $R$ is equivalent to the following statement. Every finitely generated projective $R$-module $P$ whose class in $K_{0}(R)$ is zero is already the trivial module, i.e., $0=[P] \in K_{0}(R)$ implies $P \cong 0$.

Theorem 5.3. (i) If $F$ is a field of characteristic zero, then $F G$ is stably finite for every group $G$;

(ii) If $R$ is a skew-field and $G$ is a sofic group, then $R G$ is stably finite.

Proof. [i) This is proved by Kaplansky [36] (see also Passman [51, Corollary 1.9 on page 38$]$ ).

(ii) This is proved for free-by-amenable groups by Ara-Meara-Perera [2] and extended to sofic groups by Elek-Szabo [25, Corollary 4.7]. 
Lemma 5.4. Let $R$ be a ring whose idempotents are all trivial. Let $G$ be a group such that the map induced by the inclusion $i: R \rightarrow R G$

$$
i_{*}: K_{0}(R) \otimes_{\mathbb{Z}} \mathbb{Q} \rightarrow K_{0}(R G) \otimes_{\mathbb{Z}} \mathbb{Q}
$$

is bijective and $R G$ is stably finite.

Then all idempotents in $R G$ are trivial.

Proof. Let $p$ be an idempotent in $R G$. We want to show $p \in\{0,1\}$. Denote by $\epsilon: R G \rightarrow R$ the augmentation homomorphism sending $\sum_{g \in G} r_{g} \cdot g$ to $\sum_{g \in G} r_{g}$. Since $\epsilon(p) \in R$ is 0 or 1 by assumption, it suffices to show $p=0$ under the assumption that $\epsilon(p)=0$. Let $(p) \subseteq R G$ be the ideal generated by $p$ which is a finitely generated projective $R G$-module. Since $i_{*}: K_{0}(R) \otimes_{\mathbb{Z}} \mathbb{Q} \rightarrow K_{0}(R G) \otimes_{\mathbb{Z}} \mathbb{Q}$ is surjective by assumption, we can find a finitely generated projective $R$-module $P$ and integers $k, m, n \geq 0$ satisfying

$$
(p)^{k} \oplus R G^{m} \cong_{R G} i_{*}(P) \oplus R G^{n} .
$$

If we now apply $i_{*} \circ \epsilon_{*}$ and use $\epsilon \circ i=\mathrm{id}, i_{*} \circ \epsilon_{*}\left(R G^{l}\right) \cong R G^{l}$ and $\epsilon(p)=0$ we obtain

$$
R G^{m} \cong i_{*}(P) \oplus R G^{n} .
$$

Inserting this in the first equation yields

$$
(p)^{k} \oplus i_{*}(P) \oplus R G^{n} \cong i_{*}(P) \oplus R G^{n}
$$

and therefore $0=\left[(p)^{k}\right] \in K_{0}(R G)$. Using Remark 5.2 we conclude $(p)^{k}=0$ and hence $p=0$.

Now Theorem 0.12 follows from Theorem 5.3 and Lemma 5.4

\section{Nil-groups}

In this section we give a brief discussion about Waldhausen's Nil-groups.

\subsection{Applications of homotopy $\boldsymbol{K}$-theory to Waldhausen's Nil-}

groups. The Nil-groups due to Bass $N K_{n}(R)$ have been generalized to Waldhausen's Nil groups as follows. These groups were defined by Waldhausen (see 64, [65]) for $n \geq 0$ and their extensions to $n \in \mathbb{Z}$ is explained in [4, Section 9]. For more details we refer to these papers. There are two kinds of Nil-groups, the first one taking care of amalgamated products and the second of generalized Laurent extensions.

We begin with a discussion of the one for amalgamated products. Let $\alpha: C \rightarrow A$ and $\beta: C \rightarrow B$ be pure and free and write $A=\alpha(C) \oplus A^{\prime}$ and $B=\beta(C) \oplus B^{\prime}$ and put $R=A *_{C} B$. Then the Nil-groups of the first kind are denoted by 
$\mathrm{Nil}_{n}\left(C ; A^{\prime}, B^{\prime}\right)$. The group $\operatorname{Nil}_{n}\left(C ; A^{\prime}, B^{\prime}\right)$ is a direct summand in $K_{n+1}(R)$ and there is a long exact sequence

$$
\begin{array}{r}
\cdots \rightarrow K_{n+1}(R C) \rightarrow K_{n+1}(A) \oplus K_{n+1}(B) \rightarrow K_{n+1}(R) / \mathrm{Nil}_{n}\left(C ; A^{\prime}, B^{\prime}\right) \\
\rightarrow K_{n}(C) \rightarrow K_{n}(A) \oplus K_{n}(B) \rightarrow \cdots .
\end{array}
$$

There is an analogous long exact sequence for homotopy $K$-theory which does not involve Nil-terms (see [4, Section 9])

$$
\begin{aligned}
\cdots \rightarrow K H_{n+1}(R C) \rightarrow K H_{n+1}(A) \oplus K H_{n+1}(B) \rightarrow K H_{n+1}(R) & \\
& \rightarrow K H_{n}(C) \rightarrow K H_{n}(A) \oplus K H_{n}(B) \rightarrow \cdots .
\end{aligned}
$$

The natural transformation from algebraic $K$-theory to homotopy $K$-theory induces a map between the long exact sequences (6.1) and (6.2). Now suppose that for $\mathbb{Z} \subseteq \Lambda \subseteq \mathbb{Q}$ the maps

$$
\begin{aligned}
& K_{n}(A) \otimes_{\mathbb{Z}} \Lambda \rightarrow K H_{n}(A) \otimes_{\mathbb{Z}} \Lambda ; \\
& K_{n}(B) \otimes_{\mathbb{Z}} \Lambda \rightarrow K H_{n}(B) \otimes_{\mathbb{Z}} \Lambda ; \\
& K_{n}(C) \otimes_{\mathbb{Z}} \Lambda \rightarrow K H_{n}(C) \otimes_{\mathbb{Z}} \Lambda ; \\
& K_{n}(R) \otimes_{\mathbb{Z}} \Lambda \rightarrow K H_{n}(R) \otimes_{\mathbb{Z}} \Lambda,
\end{aligned}
$$

are bijective for all $n \in \mathbb{Z}$. Then a Five-Lemma argument implies

$$
\operatorname{Nil}\left(A ; B^{\prime}, C^{\prime}\right) \otimes_{\mathbb{Z}} \Lambda=0 \quad \text { for all } n \in \mathbb{Z} .
$$

Recall from the spectral sequence of [67, Theorem 1.3] that $K_{n}(A) \otimes_{\mathbb{Z}} \Lambda \rightarrow$ $K H_{n}(A) \otimes_{\mathbb{Z}} \Lambda$ is bijective if we have $N K_{n}\left(A\left[x_{1}, x_{2}, \ldots, x_{k}\right]\right) \otimes_{\mathbb{Z}} \Lambda=0$ for all $n \in \mathbb{Z}$ and $k \in \mathbb{Z}, k \geq 0$ and analogous for $B, C$ and $R$ instead of $A$.

Next we deal with generalized Laurent extensions. Let $R$ be the generalized Laurent extension of pure and free ring maps $\alpha, \beta: C \rightarrow A$. Associated to it is the Nil-term $\operatorname{Nil}_{n}\left(C ;{ }_{\alpha} A_{\alpha}^{\prime},{ }_{\beta} A_{\beta}^{\prime \prime},{ }_{\beta} A_{\alpha},{ }_{\alpha} A_{\beta}\right)$ which is a direct summand in $K_{n+1}(R)$. We obtain long exact sequences

$$
\begin{array}{r}
\cdots \rightarrow K_{n+1}(C) \stackrel{\alpha_{*}-\beta_{*}}{\longrightarrow} K_{n+1}(A) \rightarrow K_{n+1}(R) / \mathrm{Nil}_{n}\left(C ;{ }_{\alpha} A_{\alpha}^{\prime},{ }_{\beta} A_{\beta}^{\prime \prime},{ }_{\beta} A_{\alpha},{ }_{\alpha} A_{\beta}\right) \\
\rightarrow K_{n}(C) \stackrel{\alpha_{*}-\beta_{*}}{\longrightarrow} K_{n}(A) \rightarrow \cdots
\end{array}
$$

and

$$
\begin{aligned}
\cdots \rightarrow K H_{n+1}(C) \stackrel{\alpha_{*}-\beta_{*}}{\longrightarrow} K H_{n+1}(A) & \rightarrow K H_{n+1}(R) \\
& \rightarrow K H_{n}(C) \stackrel{\alpha_{*}-\beta_{*}}{\longrightarrow} K H_{n}(A) \rightarrow \cdots .
\end{aligned}
$$

If for $\mathbb{Z} \subseteq \Lambda \subseteq \mathbb{Q}$ the maps

$$
\begin{aligned}
& K_{n}(A) \otimes_{\mathbb{Z}} \Lambda \rightarrow K H_{n}(A) \otimes_{\mathbb{Z}} \Lambda ; \\
& K_{n}(C) \otimes_{\mathbb{Z}} \Lambda \rightarrow K H_{n}(C) \otimes_{\mathbb{Z}} \Lambda ; \\
& K_{n}(R) \otimes_{\mathbb{Z}} \Lambda \rightarrow K H_{n}(R) \otimes_{\mathbb{Z}} \Lambda,
\end{aligned}
$$


are bijective for all $n \in \mathbb{Z}$, then we conclude as above that

$$
\operatorname{Nil}_{n}\left(C ;{ }_{\alpha} A_{\alpha}^{\prime},{ }_{\beta} A_{\beta}^{\prime \prime},{ }_{\beta} A_{\alpha},{ }_{\alpha} A_{\beta}\right) \otimes_{\mathbb{Z}} \Lambda=0 \quad \text { for all } n \in \mathbb{Z}
$$

holds.

We can now prove Theorem 0.15 .

Proof. (i) Because $G, H$ and $K$ are finite we conclude from Lemma 2.11)(ii) that the maps

$$
\begin{aligned}
& K_{n}(\mathbb{Z} G) \otimes_{\mathbb{Z}} \mathbb{Q} \rightarrow K H_{n}(\mathbb{Z} G) \otimes_{\mathbb{Z}} \mathbb{Q} \\
& K_{n}(\mathbb{Z} H) \otimes_{\mathbb{Z}} \mathbb{Q} \rightarrow K H_{n}(\mathbb{Z} H) \otimes_{\mathbb{Z}} \mathbb{Q} ; \\
& K_{n}(\mathbb{Z} K) \otimes_{\mathbb{Z}} \mathbb{Q} \rightarrow K H_{n}(\mathbb{Z} K) \otimes_{\mathbb{Z}} \mathbb{Q},
\end{aligned}
$$

are bijective. Let $\Gamma:=G *_{K} H$ be the amalgamated product. Then $\Gamma$ acts properly and cocompactly on a tree and is therefore word-hyperbolic. Theorems 0.1 (i) and 0.3 (i) imply $\Gamma \in \mathcal{F} \mathcal{J}(\mathbb{Z})$ and $\Gamma \in \mathcal{F J}_{K H}(\mathbb{Z})$. Consider the following commutative diagram

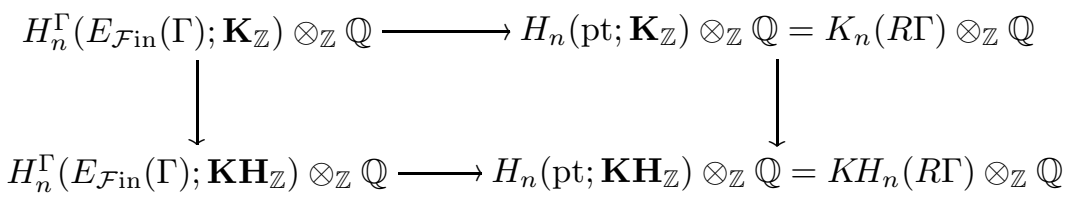

where the horizontal maps are the assembly maps induced by the projection $E_{\mathcal{F} \text { in }}(\Gamma) \rightarrow$ pt and the vertical maps are induced by the passage from algebraic $K$-theory to homotopy $K$-theory. The lower horizontal map is an isomorphism since $\Gamma \in \mathcal{F J}_{K H}(\mathbb{Z})$, the upper horizontal map is an isomorphism by Lemma 2.8 since $\Gamma \in \mathcal{F} \mathcal{J}(\mathbb{Z})$. The left vertical map is an isomorphism by Lemma 2.12 (ii) Therefore $K_{n}(\mathbb{Z} \Gamma) \otimes_{\mathbb{Z}} \mathbb{Q} \cong K H_{n}(\mathbb{Z} \Gamma) \otimes_{\mathbb{Z}} \mathbb{Q}$. The Five-Lemma argument from above implies now the rational vanishing of the Nil-groups as claimed.

(ii) Here we can argue as in (i) but now using the $H N N$-extension associated to the inclusions $\alpha$ and $\beta$ instead of the amalgamated product.

6.2. Nil-groups and rings with finite characteristic. For this subsection fix an integer $N \geq 2$ and a ring $A$ of characteristic $N$. In Lemma 2.10 we have shown that we get $N K_{n}(A)[1 / N]=0$ for every $n \in \mathbb{Z}$. This implies by the above Five-Lemma argument for all $n \in \mathbb{Z}$ and all pure and free maps $\alpha: C \rightarrow A$ and $\beta: C \rightarrow B$ and all pure and free maps $\alpha, \beta: C \rightarrow A$ respectively

$$
\begin{aligned}
\operatorname{Nil}_{n}\left(C ; A^{\prime}, B^{\prime}\right) \otimes_{\mathbb{Z}}[1 / N] & =0 ; \\
\mathrm{Nil}_{n}\left(C ;{ }_{\alpha} A_{\alpha}^{\prime},{ }_{\beta} A_{\beta}^{\prime \prime},{ }_{\beta} A_{\alpha},{ }_{\alpha} A_{\beta}\right) \otimes_{\mathbb{Z}}[1 / N] & =0 .
\end{aligned}
$$




\subsection{Nil-groups and regular rings $R$ with $\mathbb{Q} \subset \boldsymbol{R}$.}

Conjecture 6.1. Let $R$ be a regular ring with $\mathbb{Q} \subseteq R$. Then we get for all groups $G$ and all $n \in \mathbb{Z}$

$$
N K_{n}(R G)=0
$$

and the canonical map

$$
K_{n}(R G) \rightarrow K H_{n}(R G)
$$

is bijective.

By the following discussion Conjecture 6.1 is true if $G$ belongs to the class $\mathcal{F} \mathcal{J}(R)$ appearing in Theorem 0.1 but we do not know it for all groups $G$.

If $R$ is regular then the polynomial ring $R[t]$ is regular as well. Thus if $N K_{n}(R G)=0$ holds for a fixed group $G$ and all regular rings $R$ with $\mathbb{Q} \subseteq R$, then $K_{n}(R G) \rightarrow K H_{n}(R G)$ is bijective for this fixed group $G$ and all regular rings $R$ with $\mathbb{Q} \subseteq R$. This follows from the spectral sequence appearing in $[67$, Theorem 1.3]. Using the Bass-Heller-Swan decomposition it is not hard to see that similarly, if $N K_{n}(R G)=0$ holds for all groups $G$ and a fixed regular ring $R$ with $\mathbb{Q} \subseteq R$, then $K_{n}(R G) \rightarrow K H_{n}(R G)$ is bijective for all groups $G$ and this fixed regular ring $R$ with $\mathbb{Q} \subseteq R$.

If Conjecture 6.1 is true, then also Waldhausen's Nil-groups associated to a free amalgamated product of groups $G=G_{1} *_{G_{0}} G_{1}$ or to an HNN-extension with coefficients in a regular ring $R$ with $\mathbb{Q} \subseteq R$ vanish by the general argument from Section 6.1 .

Let $\mathcal{F J}_{\text {Fin }}(R)$ be the class of those groups for which the Fibered Farrell-Jones Conjecture for algebraic $K$-theory with coefficients in $R$ is true for the family $\mathcal{F}$ in. We get $\mathcal{F J}_{\text {Fin }}(R) \subseteq \mathcal{F} \mathcal{J}(R)$ from Lemma 1.2 for all rings $R$ but $\mathcal{F} \mathcal{J}_{\text {Fin }}(\mathbb{Z}) \neq$ $\mathcal{F} \mathcal{J}(\mathbb{Z})$.

If Conjecture 6.1 holds and $R$ is a regular ring with $\mathbb{Q} \subseteq R$, then the map $H_{n}^{G}\left(X ; \mathbf{K}_{R}\right) \rightarrow H_{n}^{G}\left(X ; \mathbf{K H}_{R}\right)$ induced by the transition from $K$-theory to homotopy $K$-theory is an isomorphism for any $G$ - $C W$-complex $X$ and the assembly maps for $K$-theory and homotopy $K$-theory can be identified. Thus Conjecture 6.1 implies $\mathcal{F J}_{\text {Fin }}(R)=\mathcal{F J}_{\text {KH }}(R)$. In particular, if Conjecture 6.1 holds, then by Theorem 0.3 (vi) every virtually cyclic group satisfies the Fibered Farrell-Jones Conjecture for algebraic $K$-theory with coefficients in $R$ for the family $\mathcal{F}$ in. We omit the proof (based on the Bass-Heller-Swan formula) that Conjecture 6.1 is in fact equivalent to the statement $\mathbb{Z} \in \mathcal{F} \mathcal{J}_{\text {Fin }}(R)$ for any regular $\operatorname{ring} R$ with $\mathbb{Q} \subseteq R$.

If Conjecture 6.1 is true and $R$ is a regular ring with $\mathbb{Q} \subseteq R$, then the Transitivity Principle (see Theorem 1.4) implies that $\mathcal{F} \mathcal{J}(R)=\mathcal{F J}_{\text {Fin }}(R)$, the conclusions appearing in Theorem 0.3 are true for each group in $\mathcal{F} \mathcal{J}(R)=\mathcal{F} \mathcal{J}_{\text {Fin }}(R)$, and assertion (i)] of Theorem 0.5 is true for all groups in $\mathcal{F} \mathcal{J}_{\text {Fin }}(R)$ and in particular for all elementary amenable groups.

Let $R$ be any ring. Recall that $\mathcal{F} \mathcal{J}(R)$ contains for instance word-hyperbolic groups and virtually nilpotent groups by Theorem 0.1 but we do not know whether elementary amenable groups belong to $\mathcal{F} \mathcal{J}(R)$ and whether they satisfy assertion (i) of Theorem 0.5. Our methods do not give this conclusion since we know 
only that $G \in \mathcal{F} \mathcal{J}(R)$ implies $G \times \mathbb{Z} \in \mathcal{F} \mathcal{J}(R)$ but we would at least need $G \rtimes_{\alpha} \mathbb{Z} \in \mathcal{F} \mathcal{J}(R)$ for any automorphism $\alpha: G \rightarrow G$. More generally, it would be interesting to solve the question whether for any extensions $1 \rightarrow G \rightarrow \Gamma \rightarrow V \rightarrow 1$ for virtually cyclic $V$ and $G \in \mathcal{F} \mathcal{J}(R)$ we have $\Gamma \in \mathcal{F} \mathcal{J}(R)$ because a positive answer would imply that $\mathcal{F} \mathcal{J}(R)$ is closed under extensions.

\section{The Farrell-Jones Conjecture for $L$-theory}

In this section ring $R$ will always mean associative ring with unit and involution.

Recall that the (Fibered) Farrell-Jones Conjecture for algebraic L-theory with coefficients in $R$ for the group $G$ is the (Fibered) Isomorphism Conjecture 1.1 in the special case, where the family $\mathcal{F}$ consists of all virtually cyclic subgroups of $G$ and $\mathcal{H}_{*}^{?}$ is the equivariant homology theory $\mathcal{H}_{*}^{?}\left(-; \mathbf{L}_{R}^{\langle-\infty\rangle}\right)$ associated to the Groupoids-spectrum given by algebraic $L$-theory and $R$ as coefficient ring (see 45, Section 6]). So the Farrell-Jones Conjecture for algebraic $L$-theory with coefficients in $R$ for the group $G$ predicts that the map

$$
H_{n}^{G}\left(E_{\mathcal{V} C \text { yc }}(G), \mathbf{L}_{R}^{\langle-\infty\rangle}\right) \rightarrow L_{n}^{\langle-\infty\rangle}(R G)
$$

is bijective for all $n \in \mathbb{Z}$. The original source for (Fibered) Farrell-Jones Conjecture is 28. The corresponding conjecture is false if one replaces the decoration $\langle-\infty\rangle$ with the decoration $p, h$ or $s$ (see 31]). For the status of the Farrell-Jones Conjecture with coefficients in $\mathbb{Z}$ we refer for instance to [45. Sections 5.2 and 5.3].

The next result is proved in [3, Lemma 5.2].

Lemma 7.1. Let $R$ be a ring. Then the equivariant homology theory $\mathcal{H}_{*}^{?}\left(-; \mathbf{L}_{R}^{\langle-\infty\rangle}\right)$ is strongly continuous.

Theorem 1.7 (ii) and Lemma 7.2 imply that for any ring $R$ and any direct system of groups $\left\{G_{i} \mid i \in I\right\}$ (with not necessarily injective structure maps) $G=\operatorname{colim}_{i \in I} G_{i}$ satisfies the Fibered Farrell Jones Conjecture for algebraic $L$ theory with coefficients in $R$ if each group $G_{i}$ does.

In algebraic $L$-theory the situation simplifies if one inverts 2 .

Lemma 7.2. Let $R$ be a ring and let $G$ be a group. Then the Fibered Farrell Jones Conjecture with coefficients in $R$ after applying $-\otimes_{\mathbb{Z}} \mathbb{Z}[1 / 2]$ to the assembly map holds for $(G, \mathcal{V C} y c)$ if and only the same holds for $(G, \mathcal{F}$ in $)$.

Proof. Because of Lemma 1.10 and the fact that every virtually cyclic group maps surjectively onto $\mathbb{Z}$ or $\mathbb{Z} / 2 * \mathbb{Z} / 2$ it suffices to show the claim for $G=\mathbb{Z}$ and $G=$ $\mathbb{Z} / 2 * \mathbb{Z} / 2$. These cases follow from the exact sequence involving UNil-terms and the proof that UNil-groups are 2 -torsion due to Cappell $[18$ in the case $\mathbb{Z} / 2 * \mathbb{Z} / 2$. In the case $\mathbb{Z}$ there are no UNil-terms for infinite virtually cyclic groups of the first kind. This follows essentially from [55] and [56] as carried out in [43, Lemma 4.2].

The Transitivity Principle (see Theorem 1.4) implies that for a ring $R$ and a group $G$ the Farrell-Jones Conjecture for algebraic $L$-theory with coefficients in $R$ is 
true after applying $-\otimes_{\mathbb{Z}} \mathbb{Z}[1 / 2]$ to the assembly map for the family $\mathcal{V C}$ yc if and only if this is true for the family $\mathcal{F}$ in. This implies that assertions (ii)[(iii)|(iv)] and (vi) appearing in Theorem 0.3 are true for the family $\mathcal{F J}_{L[1 / 2]}(R)$ of those groups for which the Farrell-Jones Conjecture for algebraic $L$-theory with coefficients in $R$ is true after applying $-\otimes_{\mathbb{Z}} \mathbb{Z}[1 / 2]$. We do not know whether all word hyperbolic groups belong to $\mathcal{F J}_{L[1 / 2]}(R)$. Farrell and Jones claim in [28, Remark 2.1.3] without giving details that the $L$-theory version of their celebrated [28, Theorem 1.2] is true. This together with [57, 3.6.4] implies that all virtually finitely generated abelian groups belong to $\mathcal{F} \mathcal{J}_{L[1 / 2]}(R)$ for $R=\mathbb{Z}, \mathbb{Q}$. Hence all elementary amenable groups belong to $\mathcal{F J}_{L[1 / 2]}(R)$ for $R=\mathbb{Z}, \mathbb{Q}$ by Lemma 1.12. This has already been proved in 29 , Section 5].

\section{References}

[1] G. Almkvist. Endomorphisms of finitely generated projective modules over a commutative ring. Ark. Mat., 11:263-301, 1973.

[2] P. Ara, K. C. O'Meara, and F. Perera. Stable finiteness of group rings in arbitrary characteristic. Adv. Math., 170(2):224-238, 2002.

[3] A. Bartels, S. Echterhoff, and W. Lück. Inheritance of isomorphism conjectures under colimits. Preprintreihe SFB 478 - Geometrische Strukturen in der Mathematik, Heft 452, Münster, arXiv:math.KT/0702460, 2007.

[4] A. Bartels and W. Lück. Induction theorems and isomorphism conjectures for $K$ - and $L$-theory. Preprintreihe SFB 478 - Geometrische Strukturen in der Mathematik, Heft 331, Münster, arXiv:math.KT/0404486, to appear in Forum Math., 2004.

[5] A. Bartels and W. Lück. Isomorphism conjecture for homotopy $K$-theory and groups acting on trees. J. Pure Appl. Algebra, 205(3):660-696, 2006.

[6] A. Bartels, W. Lück, and H. Reich. The $K$-theoretic Farrell-Jones Conjecture for hyperbolic groups. Preprintreihe SFB 478 - Geometrische Strukturen in der Mathematik, Heft 450, Münster, arXiv:math.KT/0701434 2007.

[7] A. Bartels and H. Reich. Coefficients for the Farrell-Jones conjecture. Preprintreihe SFB 478 - Geometrische Strukturen in der Mathematik, Heft 402, Münster, arXiv:math.KT/0510602, to appear in Advances, 2005.

[8] A. Bartels and H. Reich. On the Farrell-Jones conjecture for higher algebraic $K$ theory. J. Amer. Math. Soc., 18(3):501-545 (electronic), 2005.

[9] A. C. Bartels. On the domain of the assembly map in algebraic K-theory. Algebr. Geom. Topol., 3:1037-1050 (electronic), 2003.

[10] H. Bass. Algebraic K-theory. W. A. Benjamin, Inc., New York-Amsterdam, 1968.

[11] H. Bass. Traces and Euler characteristics. In Homological group theory (Proc. Sympos., Durham, 1977), volume 36 of London Math. Soc. Lecture Note Ser., pages 1-26. Cambridge Univ. Press, Cambridge, 1979.

[12] A. J. Berrick, I. Chatterji, and G. Mislin. From acyclic groups to the Bass conjecture for amenable groups. Math. Ann., 329(4):597-621, 2004.

[13] A. J. Berrick, I. Chatterji, and G. Mislin. Homotopy idempotents on manifolds and Bass' conjectures. Geometry and Topology Monographs, 10:41-62, 2007. 
[14] M. Bridson. Non-positive curvature and complexity for finitely presented groups. lecture on the ICM 2006 in Madrid, to appear in the ICM-Proceedings, 2006.

[15] M. R. Bridson and C. F. Miller, III. Recognition of subgroups of direct products of hyperbolic groups. Proc. Amer. Math. Soc., 132(1):59-65 (electronic), 2004.

[16] K. S. Brown. Cohomology of groups, volume 87 of Graduate Texts in Mathematics. Springer-Verlag, New York, 1982.

[17] M. Burger and A. Valette. Idempotents in complex group rings: theorems of Zalesskii and Bass revisited. J. Lie Theory, 8(2):219-228, 1998.

[18] S. E. Cappell. Unitary nilpotent groups and Hermitian K-theory. I. Bull. Amer. Math. Soc., 80:1117-1122, 1974.

[19] G. Cliff and A. Weiss. Moody's induction theorem. Illinois J. Math., 32(3):489-500, 1988.

[20] J. F. Davis and W. Lück. Spaces over a category and assembly maps in isomorphism conjectures in $K$ - and $L$-theory. $K$-Theory, 15(3):201-252, 1998.

[21] T. Delzant. Sur l'anneau d'un groupe hyperbolique. C. R. Acad. Sci. Paris Sér. I Math., 324(4):381-384, 1997.

[22] C. Deninger. p-adic entropy and a $p$-adic Fuglede-Kadison determinant. Preprint, 2006.

[23] B. Eckmann. Cyclic homology of groups and the Bass conjecture. Comment. Math. Helv., 61(2):193-202, 1986.

[24] B. Eckmann. Projective and Hilbert modules over group algebras, and finitely dominated spaces. Comment. Math. Helv., 71(3):453-462, 1996.

[25] G. Elek and E. Szabó. Sofic groups and direct finiteness. J. Algebra, 280(2):426-434, 2004.

[26] G. Elek and E. Szabó. On sofic groups. J. Group Theory, 9(2):161-171, 2006.

[27] I. Emmanouil. On a class of groups satisfying Bass' conjecture. Invent. Math., 132(2):307-330, 1998.

[28] F. T. Farrell and L. E. Jones. Isomorphism conjectures in algebraic $K$-theory. $J$. Amer. Math. Soc., 6(2):249-297, 1993.

[29] F. T. Farrell and P. A. Linnell. K-theory of solvable groups. Proc. London Math. Soc. (3), 87(2):309-336, 2003.

[30] F. T. Farrell and P. A. Linnell. Whitehead groups and the Bass conjecture. Math. Ann., 326(4):723-757, 2003.

[31] T. Farrell, L. Jones, and W. Lück. A caveat on the isomorphism conjecture in L-theory. Forum Math., 14(3):413-418, 2002.

[32] E. Formanek. Idempotents in Noetherian group rings. Canad. J. Math., 25:366-369, 1973.

[33] M. Gromov. Spaces and questions. Geom. Funct. Anal., special volume(Special Volume, Part I):118-161, 2000. GAFA 2000 (Tel Aviv, 1999).

[34] J. Grunewald. The behaviour of nil-groups under localization. arXiv:math.KT/0005194, 2006.

[35] N. Higson, V. Lafforgue, and G. Skandalis. Counterexamples to the Baum-Connes conjecture. Geom. Funct. Anal., 12(2):330-354, 2002. 
[36] I. Kaplansky. Fields and rings. The University of Chicago Press, Chicago, Ill.London, 1969.

[37] V. Lafforgue. Une démonstration de la conjecture de Baum-Connes pour les groupes réductifs sur un corps $p$-adique et pour certains groupes discrets possédant la propriété (T). C. R. Acad. Sci. Paris Sér. I Math., 327(5):439-444, 1998.

[38] P. A. Linnell. Decomposition of augmentation ideals and relation modules. Proc. London Math. Soc. (3), 47(1):83-127, 1983.

[39] W. Lück. Dimension theory of arbitrary modules over finite von Neumann algebras and $L^{2}$-Betti numbers. II. Applications to Grothendieck groups, $L^{2}$-Euler characteristics and Burnside groups. J. Reine Angew. Math., 496:213-236, 1998.

[40] W. Lück. Chern characters for proper equivariant homology theories and applications to $K$ - and L-theory. J. Reine Angew. Math., 543:193-234, 2002.

[41] W. Lück. $L^{2}$-invariants: theory and applications to geometry and $K$-theory, volume 44 of Ergebnisse der Mathematik und ihrer Grenzgebiete. 3. Folge. A Series of Modern Surveys in Mathematics [Results in Mathematics and Related Areas. 3rd Series. A Series of Modern Surveys in Mathematics]. Springer-Verlag, Berlin, 2002.

[42] W. Lück. The relation between the Baum-Connes conjecture and the trace conjecture. Invent. Math., 149(1):123-152, 2002.

[43] W. Lück. $K$ - and $L$-theory of the semi-direct product of the discrete 3 -dimensional Heisenberg group by $\mathbb{Z} / 4$. Geom. Topol., 9:1639-1676 (electronic), 2005.

[44] W. Lück. Survey on classifying spaces for families of subgroups. In Infinite groups: geometric, combinatorial and dynamical aspects, volume 248 of Progr. Math., pages 269-322. Birkhäuser, Basel, 2005.

[45] W. Lück and H. Reich. The Baum-Connes and the Farrell-Jones conjectures in $K$ and $L$-theory. In Handbook of K-theory. Vol. 1, 2, pages 703-842. Springer, Berlin, 2005.

[46] W. Lück and H. Reich. Detecting K-theory by cyclic homology. Proc. of the LMS, 93(3):593-634, 2006.

[47] W. Lück, H. Reich, J. Rognes, and M. Varisco. Algebraic $K$-theory of integral group rings and topological cyclic homology. in preparation, 2007.

[48] I. Mineyev and G. Yu. The Baum-Connes conjecture for hyperbolic groups. Invent. Math., 149(1):97-122, 2002.

[49] J. A. Moody. Induction theorems for infinite groups. Bull. Amer. Math. Soc. (N.S.), 17(1):113-116, 1987.

[50] J. A. Moody. Brauer induction for $G_{0}$ of certain infinite groups. J. Algebra, 122(1):114, 1989.

[51] D. S. Passman. The algebraic structure of group rings. Wiley-Interscience [John Wiley \& Sons], New York, 1977. Pure and Applied Mathematics.

[52] M. Puschnigg. The Kadison-Kaplansky conjecture for word-hyperbolic groups. Invent. Math., 149(1):153-194, 2002.

[53] D. Quillen. Higher algebraic K-theory. I. In Algebraic K-theory, I: Higher K-theories (Proc. Conf., Battelle Memorial Inst., Seattle, Wash., 1972), pages 85-147. Lecture Notes in Math., Vol. 341. Springer-Verlag, Berlin, 1973. 
[54] F. Quinn. Hyperelementary assembly for $k$-theory of virtually abelain groups. preprint, arXiv:math.KT/0509294, 2005.

[55] A. A. Ranicki. Algebraic L-theory. II. Laurent extensions. Proc. London Math. Soc. (3), 27:126-158, 1973.

[56] A. A. Ranicki. Algebraic L-theory. III. Twisted Laurent extensions. In Algebraic Ktheory, III: Hermitian K-theory and geometric application (Proc. Conf. Seattle Res. Center, Battelle Memorial Inst., 1972), pages 412-463. Lecture Notes in Mathematics, Vol. 343. Springer, Berlin, 1973.

[57] A. A. Ranicki. Exact sequences in the algebraic theory of surgery. Princeton University Press, Princeton, N.J., 1981.

[58] S. Roushon. The Farrell-Jones isomorphism conjecture for 3-manifold groups. arXiv:math.KT/0405211 4 , 2006.

[59] J. A. Schafer. The Bass conjecture and group von Neumann algebras. K-Theory, 19(3):211-217, 2000.

[60] J.-P. Serre. Linear representations of finite groups. Springer-Verlag, Berlin, 1997.

[61] G. Skandalis. Progrès récents sur la conjecture de Baum-Connes. Contribution de Vincent Lafforgue. Astérisque, 276:105-135, 2002. Séminaire Bourbaki, Vol. $1999 / 2000$.

[62] J. Stienstra. Operations in the higher $K$-theory of endomorphisms. In Current trends in algebraic topology, Part 1 (London, Ont., 1981), volume 2 of CMS Conf. Proc., pages 59-115. Amer. Math. Soc., Providence, R.I., 1982.

[63] R. G. Swan. Induced representations and projective modules. Ann. of Math. (2), $71: 552-578,1960$.

[64] F. Waldhausen. Algebraic $K$-theory of generalized free products. I, II. Ann. of Math. (2), 108(1):135-204, 1978.

[65] F. Waldhausen. Algebraic $K$-theory of topological spaces. I. In Algebraic and geometric topology (Proc. Sympos. Pure Math., Stanford Univ., Stanford, Calif., 1976), Part 1, pages 35-60. Amer. Math. Soc., Providence, R.I., 1978.

[66] C. A. Weibel. Mayer-Vietoris sequences and module structures on $N K_{*}$. In Algebraic K-theory, Evanston 1980 (Proc. Conf., Northwestern Univ., Evanston, Ill., 1980), volume 854 of Lecture Notes in Math., pages 466-493. Springer, Berlin, 1981.

[67] C. A. Weibel. Homotopy algebraic K-theory. In Algebraic K-theory and algebraic number theory (Honolulu, HI, 1987), volume 83 of Contemp. Math., pages 461-488. Amer. Math. Soc., Providence, RI, 1989.

Arthur Bartels, Mathematisches Institut, Westfälische Wilhelms-Universität Münster, Einsteinstr. 62, D-48149 Münster, Germany

E-mail: bartelsa@math.uni-muenster.de

Wolfgang Lück, Mathematisches Institut, Westfälische Wilhelms-Universität Münster, Einsteinstr. 62, D-48149 Münster, Germany

E-mail: lueck@math.uni-muenster.de

Holger Reich, Mathematisches Institut, Heinrich-Heine-Universität Düsseldorf, Universitätsstr. 1, D-40225 Düsseldorf, Germany

E-mail: holger.reich@googlemail.com 